\title{
Maps, Coordinate Reference Systems and Visualising Geographic Data with mapmisc
}

by Patrick E. Brown

\begin{abstract}
The mapmisc package provides functions for visualising geospatial data, including fetching background map layers, producing colour scales and legends, and adding scale bars and orientation arrows to plots. Background maps are returned in the coordinate reference system of the dataset supplied, and inset maps and direction arrows reflect the map projection being plotted. This is a "light weight" package having an emphasis on simplicity and ease of use.
\end{abstract}

\section{Introduction}

$\mathrm{R}$ has extensive facilities for managing, manipulating, and visualising spatial data, with the sp (Pebesma and Bivand, 2005) and raster (Hijmans, 2015b) packages providing a set of object classes and core functions which numerous other packages have built on. It is fairly straightforward to import spatial data of a variety of types and from a range of sources including: images for map backgrounds; high-resolution pixel grids of surface elevation; and polygons of administrative region boundaries. Large volumes of such data are available for download from sites such as worldgrids.org, gadm.org, and nhgis.org, and map images are freely available from OpenStreetMap.org and other online maps. The first issue often encountered after downloading and importing spatial data is reconciling different coordinate reference systems (CRS's, or map projections). Most repositories of spatial data provide longitude-latitude coordinates, although single-country data sources often use a country-specific map projection (i.e. the UK's Ordinance Survey National Grid) and online maps mostly use the Web Mercator projection. The suitability of a particular map projection will depend on the geographic region being considered and the specific problem at hand.

The mapmisc package (Brown, 2016) provides tools for working with projected data which cover the following four areas:

- producing maps with projected data, including scale bars, background images, and inset maps;

- defining and using equal-area map projections for displaying the entire globe;

- creating optimal region-specific map projections where distances are preserved; and

- mapping with colour scales for continuous and categorical data.

This paper will cover each of these points in turn, working through examples and briefly describing the operations by the functions in the mapmisc package. An emphasis is given to tidy, intuitive, and reproducible code accessible for students and non-specialists.

\section{Installation and related packages}

The two most important packages required for using spatial data in $\mathrm{R}$ are the sp and raster packages, which provide tools and classes for vector data (spatial data on a continuous domain) and raster data (defined on a pixelated grid) respectively. Installing mapmisc with install. packages ("mapmisc") or by using a menu item on a GUI will install sp and raster if they are not already present. A third important spatial package is rgdal (Bivand et al., 2016), which provides methods for re-projecting coordinates and importing spatial data in various file formats. The Geographic Data Abstraction Language (GDAL) underlies rgdal, aligning with R's UNIX-like philosophy of combining separate and specialised pieces of software. On most UNIX-based systems, the GDAL and proj4 software must be installed separately prior to installing rgdal. All versions of Windows and most versions of MacOS have binary versions of rgdal which include the GDAL and proj4 binaries, and rgdal can be installed in the same manner as any other $\mathrm{R}$ package.

Additional packages used by mapmisc are: RColorBrewer (Neuwirth, 2014), classInt (Bivand, 2015), rgeos (Bivand and Rundel, 2016), and geosphere (Hijmans, 2015a). These four packages and rgdal are not always installed automatically with mapmisc, as they are marked as "suggested" packages with mapmisc being usable with a reduced level of functionality without them. Three further packages necessary for reproducing the examples in this paper are dismo (Hijmans et al., 2016), maptools (Bivand and Lewin-Koh, 2016), and R.utils (Bengtsson, 2016).

Loading the packages with 
library("rgdal")

library ("mapmisc")

also makes sp and raster available. The remaining packages do not need to be loaded explicitly and will be called by mapmisc as needed.

\section{Getting started with spatial data in $\mathbf{R}$}

The getData function provided by raster is able to download a number of useful and interesting spatial datasets. The coastline and borders of Finland can be fetched with

finland <- raster: :getData("GADM", country = "FIN", level = 0 )

The object finland is a "SpatialPolygonsDataFrame", and Bivand et al. (2013) contains a wealth of information on working with objects of this type. The command

plot (finland, axes $=$ TRUE)

produces the plot in Figure 1a.

The choice of Finland as an example is due to its being far from the equator, and a useful contrast on the other side of the world is New Zealand. The coastline of New Zealand, obtained with

nz <- raster: :getData("GADM", country = "NZL", level = 0 )

includes a number of small outlying islands. The spatial extent of the nz object,

$\begin{array}{ll}\text { raster: : extent }(n z) \\ \text { \#\# class } & : \text { Extent } \\ \text { \#\# xmin } & :-179 \\ \text { \#\# xmax } & : 179 \\ \text { \#\# ymin } & :-52.6 \\ \text { \#\# ymax } & :-29.2\end{array}$

spans the entire globe in the $x$-direction since New Zealand has islands on both sides of the $180^{\circ}$ meridian. Finding an appropriate axis limit through trial and error brings one to

$\operatorname{plot}(n z, x \lim =c(167,178)$, axes $=$ TRUE $)$

and the map in Figure $1 b$.

The outlying islands can be removed from the nz object using the crop function in the raster package, which in turn calls rgeos. Using the locator function and a few iterations of trial and error leads to the discovery that a region spanning 160 to 180 degrees longitude and -47 to -30 degrees latitude boxes in the main islands of New Zealand fairly tightly. The parts of New Zealand contained within this box can be extracted by creating an extent object and passing it to crop.

nzClip <- raster: $: \operatorname{crop}(n z, \operatorname{extent}(160,180,-47,-30))$

The finland and nzClip objects will be used in the mapping examples which follow.

\section{Working with map projections}

This section covers mapping projected data and defining customised map projections. Adding background images, scale bars, and inset maps to plots with the map. new, openmap, scaleBar, and insetMap functions is demonstrated in the production of Figure 2. Map projections suitable for displaying the entire globe are constructed with the moll function, and along with the wrapPolys function Figure 5 is made. Map projections where Euclidean distances from $x-y$ coordinates are useful approximations of shortest distances between points on the globe are obtained with the omerc function and used to produce Figure 7.

\section{Spatial data with coordinate reference systems}

The spatial coordinates in Figures $1 \mathrm{a}$ and $1 \mathrm{~b}$ are angles of longitudes and latitudes; coordinates which would be equivalent to the two angles of the spherical coordinate system $(\rho, \theta, \phi)$ familiar to mathematicians were it not for the inconvenient fact that the Earth is not spherical. The Earth is rather an oblate spheroid, slightly "squashed" or pumpkin-shaped, and the angles of orientations of lines pointing directly "up" (with reference to the stars) and "down" (as defined by a plumb line 
pulled straight by gravity) differ. As a result, various types of long-lat coordinates are in use, with the World Geodesic System (WGS84) used by Global Positioning Systems being the most widespread. The European Petroleum Standards Group (EPSG) catalogue of Coordinate Reference Systems (or CRS's) refers to this system by the code 4326 , and this code can be used to create an R object of class "CRS" corresponding to the WGS84 system using the CRS function from the sp package.

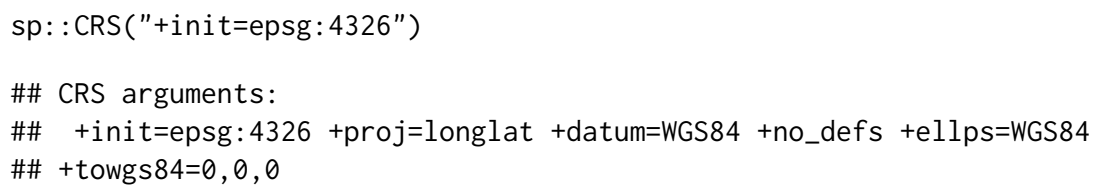

The syntax of +argument=value for specifying a CRS comes from the PROJ.4 Cartographic Projections Library, with +proj=longlat indicating coordinates are angles and ellps=WGS84 specifying that the Earth is an ellipsoid with values of the major axis length, minor axis length, and flattening corresponding to the WGS84 specification.

The difficulty angular coordinates pose for interpreting maps or using spatial statistics is that Euclidean distance $\sqrt{x^{2}+y^{2}}$ is not always a useful measure of the distance separating two points. The shortest route between two points on a sphere follows a Great Circle which divides the globe into two equal halves. The distance between two points along this path, the Great Circle distance (see Wikipedia, 2015a), can be computed with a trigonometric formula as implemented in the spDists function in sp. Euclidean distance will be roughly proportional to Great Circle distance for two points near the equator and reasonably close to one another. In Finland and New Zealand, however, Euclidean distance will over-emphasise the east-west direction since one degree of longitude is a much shorter distance (in kilometres) than one degree of latitude. It is for this reason that Greenland appears larger than India on many maps even though the opposite is true. Most R packages which perform spatial analyses rely on Euclidean distance, including this author's geostatsp (Brown, 2015), even though Great Circle distance would be straightforward to implement. Fitting a spatial model with geostatsp to data in long-lat coordinates from Finland might uncover directional effects with strong correlation in the east-west direction, which could well be an artefact arising from the over-estimation of east-west distances. The importance of transforming spherical coordinates to a coordinate system where the Euclidean distance is a reasonable approximation to the Great Circle distance should not be under-estimated.

Most countries have an "official" CRS which produces accurate Euclidean distances for specific areas of the globe, one of which is the Finland Uniform Coordinate System having EPSG code 2393. This projection is obtained in R by CRS with

CRS("+init=epsg: 2393")

\#\# CRS arguments:

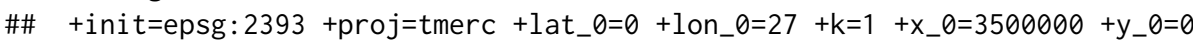

\#\# +ellps=intl +towgs $84=-96.062,-82.428,-121.753,4.801,0.345,-1.376,1.496$

\#\# +units=m +no_defs

This is a Transverse Mercator projection (+proj=tmerc) with $x$ and $y$ coordinates giving positions on a cylinder containing the earth. The entry $+\operatorname{lon}_{-} \theta=27$ indicates that the cylinder touches the Earth along the $27^{\circ}$ meridian line. Provided two points are reasonably close to the $27^{\circ}$ meridian, Euclidean distance between their Finland Uniform Coordinates will be very close to the true distance between them. The map of Finland can be converted to this coordinate system using spTransform from sp and rgdal with

finlandMerc <- spTransform(finland, CRS("+init=epsg:2393"))

Figure $1 \mathrm{c}$ is the result of plotting this object with plot(finlandMerc, axes = TRUE). Notice the projected map has a wider base and narrower top than the long-lat map in Figure 1a. The coordinates in Figure $1 \mathrm{c}$ refer to an origin where the $27^{\circ}$ meridian intersects the equator, with the $+x_{-} \theta=$ argument above indicating that $3,500 \mathrm{~km}$ are added to the $x$ coordinates.

Cylindrical map projections can be constructed from any cylinder containing the Earth, and there is no mathematical requirement to use one of the standard transverse Mercator projections with an EPSG number. For example, a given user might consider the point $\left(170^{\circ} \mathrm{E}, 45^{\circ} \mathrm{S}\right)$ to be an intuitive location for the origin of the transformed map of New Zealand, and thus decide to define a custom CRS with this centre. The cylinder can follow any Great Circle, it need not be a meridian line, and a Great Circle angled $40^{\circ}$ clockwise would run the length of the two islands. Cylindrical projections with an angle are termed Oblique Mercator projections (see Snyder, 1987, page 66), and can be constructed with the assistance of mapmisc's omerc function. A custom projection with the $\left(170^{\circ} \mathrm{E}, 45^{\circ} \mathrm{S}\right)$ origin and $40^{\circ}$ rotation is obtained by 


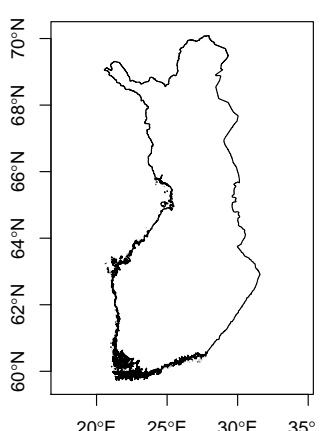

(a) Finland, Longitude-Latitude

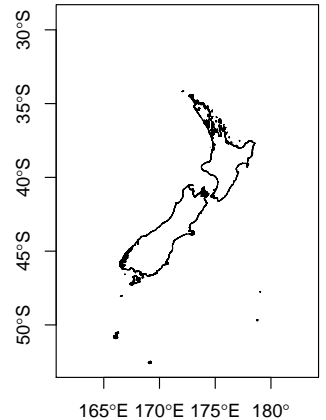

(b) New Zealand, Longitude-Latitude

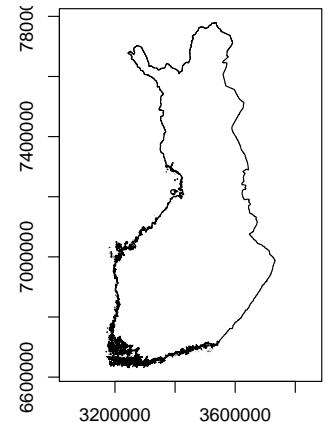

(c) Finland Transverse Mercator

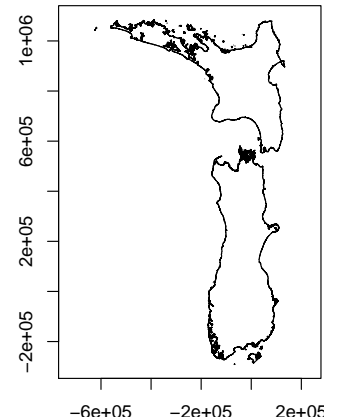

(d) New Zealand, Oblique Mercator

Figure 1: Basic maps of Finland and New Zealand in different Coordinate Reference Systems.

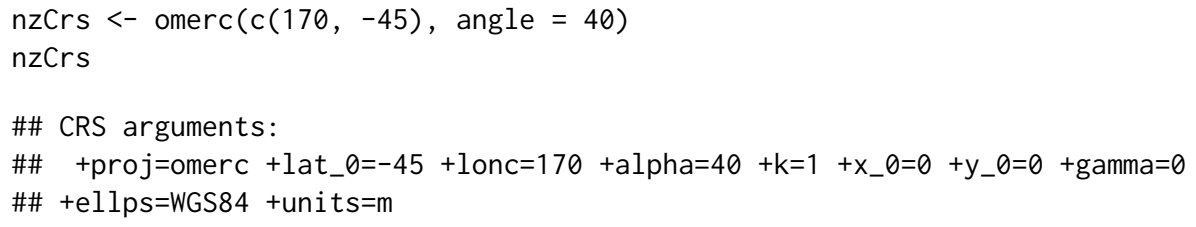

The difference between the above and the projection for Finland is the omerc in place of tmerc, with the additional argument $+a l p h a=40$ specifying an angle. The New Zealand coastline can be projected to this CRS with spTransform.

nzRot $<-$ spTransform(nzClip, nzCrs)

Figure $1 \mathrm{~d}$ results from executing plot ( $\mathrm{nzRot}$, axes $=$ TRUE), and New Zealand has been rotated $40^{\circ}$ to a vertical position.

\section{Finding a projection}

When choosing a map projection for a dataset, a simple web search of a phrase such as "map projection finland epsg" will often give clear advice as to what the most commonly used national CRS is. A number of tools in rgdal can be used to obtain a projection in a more systematic manner. Below the make_EPSG function creates a table of all EPSG coded CRS's which rgdal supports, and a grep command used to show all those projections with "Finland" in its description. The resulting list of projections below confirms that 2393 is a sensible choice.

allEpsg <- rgdal: :make_EPSG()

allEpsg[grep("Finland", allEpsg\$note), 1:2]

$\begin{array}{llr}\text { \#\# } & \text { code } & \text { note } \\ \text { \#\# } 859 & 2391 & \text { \# KKJ / Finland zone } 1 \\ \text { \#\# } 860 & 2392 & \text { \# KKJ / Finland zone } 2 \\ \text { \#\# } 861 & 2393 & \text { \# KKJ / Finland Uniform Coordinate System } \\ \text { \#\# } 862 & 2394 & \text { \# KKJ / Finland zone } 4 \\ \text { \#\# } 1853 \text { 3386 } & \text { \# KKJ / Finland zone } 0 \\ \text { \#\# } 18543387 & \text { \# KKJ / Finland zone } 5 \\ \text { \#\# } 4929 \text { 3901 \# KKJ / Finland Uniform Coordinate System + N60 height }\end{array}$

A second method for obtaining a CRS is to make a rough guess at a projection string and use showEPSG to attempt to find a corresponding EPSG code. Were one to use a Universal Transverse Mercator (or UTM) projection for a map of Finland, a web search for "UTM zone map" shows that Finland lies in UTM zone 35. A proj4 specification of a UTM zone 35 projection will contain + proj=utm and + zone $=35$, and showEPSG states that the EPSG code 32635 corresponds to an appropriate CRS.

rgdal: : showEPSG("+proj=utm +zone=35")

\#\# [1] "32635"

CRS("+init=epsg: $32635 "$ ) 


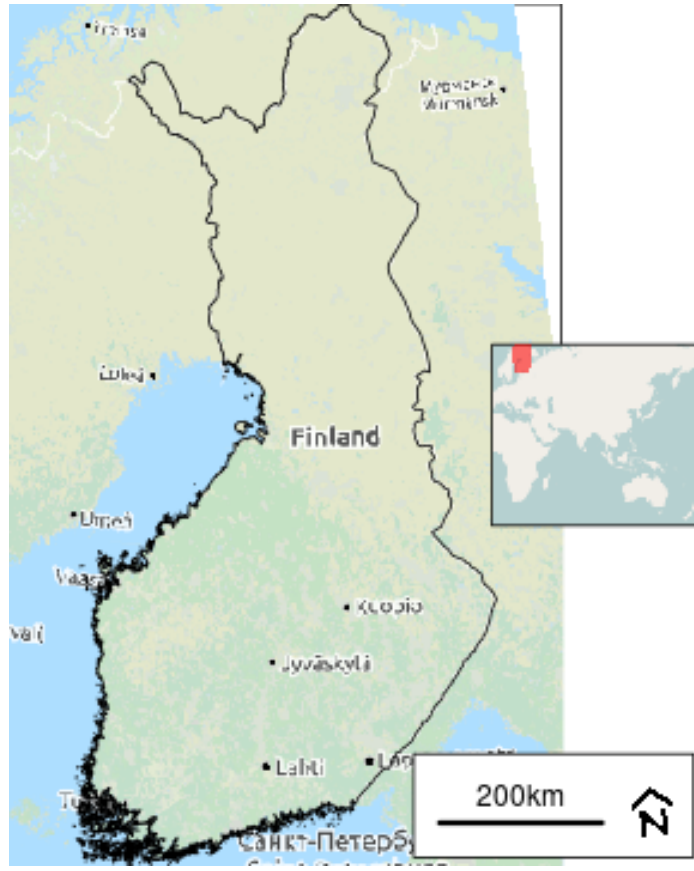

(a) Finland. Background (c) OpenStreetMap

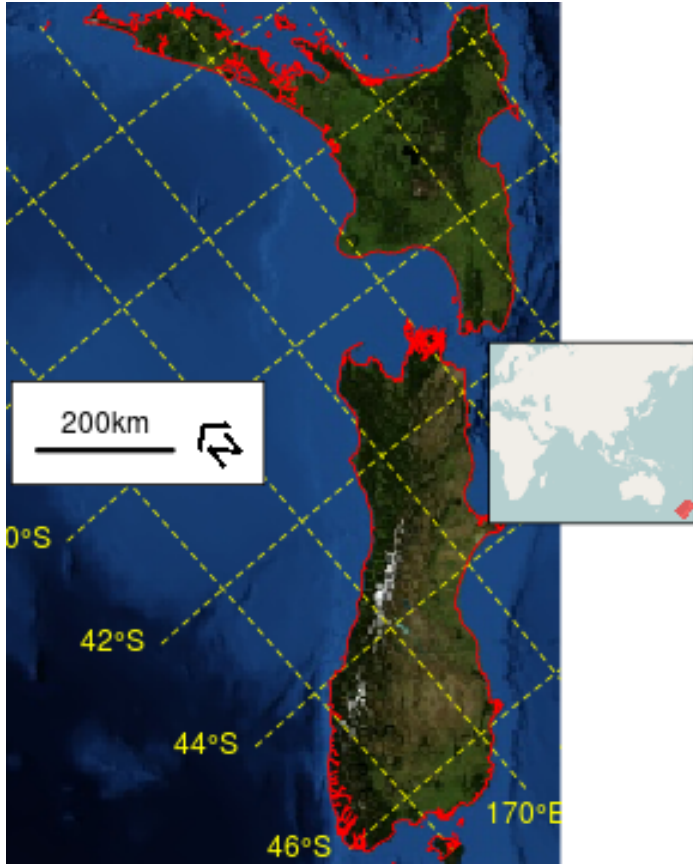

(b) New Zealand. Tiles courtesy of MapQuest and (C) OpenStreetMap

Figure 2: Maps produced using the mapmisc package, containing background images, inset maps, and scale bars.

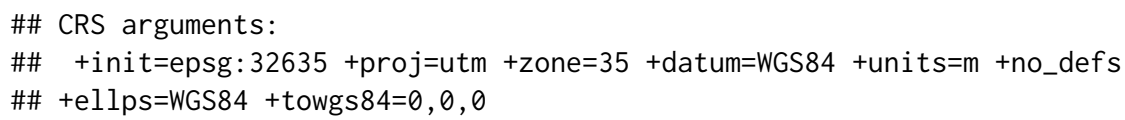

The definitive resource for information on national map projections is contained in the monthly bulletins of The Imaging and Geospatial Information Society. The GridsDatums data set in rgdal gives the year and month for each country's entry at www.asprs.org/Grids-Datums.html. The entry for Finland appears in the October 2006 issue.

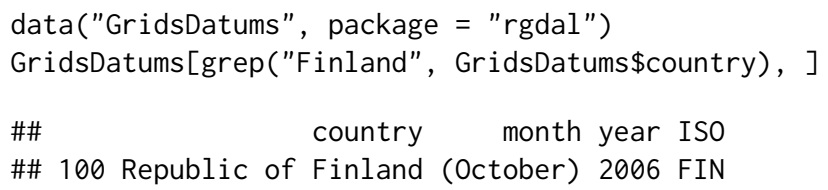

\section{Mapping projected data}

The mapmisc functions openmap, map. new, scaleBar, and insetMap can be used together to improve on the basic maps in Figure 1, and they are used here to add background images, a scale bar, and an inset map to Figure 2.

Background map images are obtained from the openmap function, which downloads image files from OpenStreetMap.org or a number of other sources. The images used in Figure 2 were obtained with

nzBg <- openmap (nzRot, path = "mapquest-sat")

finlandBg <- openmap(finlandMerc, path = "landscape")

The first argument of openmap is used to set both the spatial extent of the map to be retrieved and the CRS the map will be projected to. Any spatial object $x$ for which extent $(x)$ and projection $(x)$ are defined can be provided to openmap. The path = argument specifies the source of the map, and sample maps from the various sources are shown in Figure 11 in the Appendix. The objects produced by openmap are raster objects, converted from image files downloaded from web map servers. The Finland map is an object of class "RasterLayer".

finlandBg 


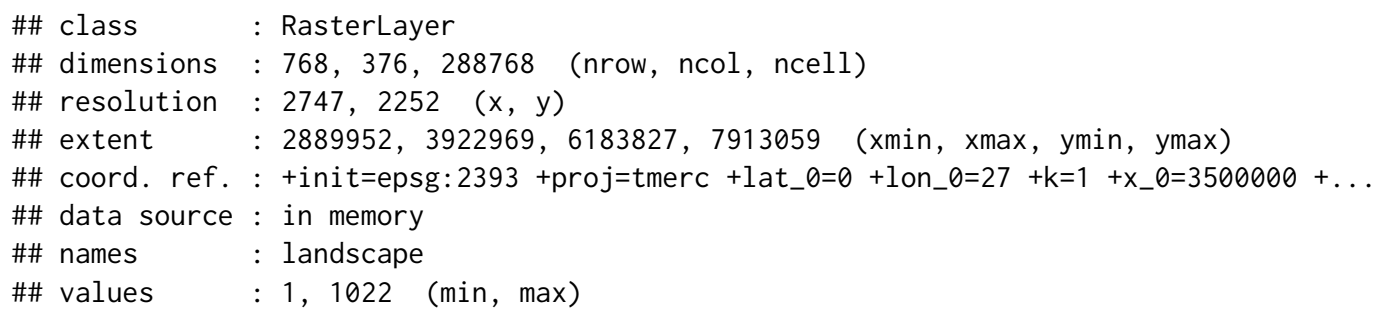

Notice that the CRS is the same as for the finlandMerc object. The New Zealand map is a "RasterStack" with red, green and blue layers.

$\mathrm{nzBg}$

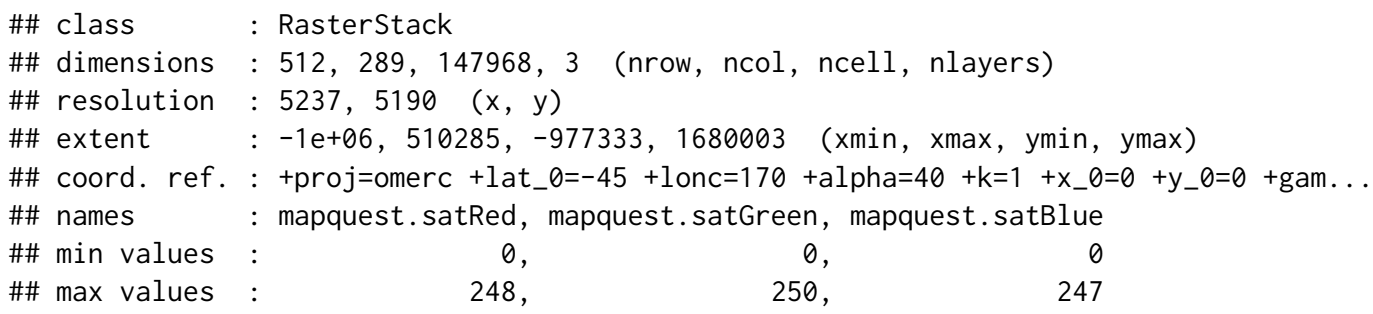

The Finland map can be viewed with plot (finlandBg), whereas the three-layered New Zealand map needs the plotRGB function for plotting its red, green and blue values as colours.

Figure $2 \mathrm{a}$ is produced with the four function calls below.

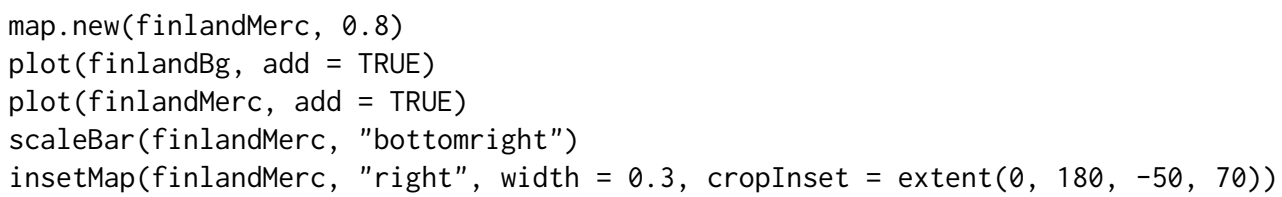

The functions run are the following:

- map. new initialises a new plot area suitable for showing finlandMerc. The second argument set to 0.8 specifies that the left $80 \%$ of the plot will contain the map and the right $20 \%$ will be reserved for legends or inset maps.

- $\operatorname{plot}($ finlandBg, add $=$ TRUE) adds the background map to the existing plot.

- $\operatorname{plot}($ finlandMerc, add = TRUE) adds the border of Finland from the finlandMerc object.

- scaleBar produces the $200 \mathrm{~km}$ scale and north arrow at the bottom right. The finlandMerc object is required to inform scaleBar that the Finland Uniform Coordinate System is used, and scaleBar (CRS ("+init=epsg:2393"), "bottomright") would have achieved the same effect.

- insetMap produces the small map to the right, showing in red on the inset map the area covered by the plot. As with scaleBar it uses finlandMerc to obtain the CRS of the map coordinates. The width argument specifies the width of the inset map as a fraction of the plotting region. The cropInset argument produces an inset map where New Zealand (at $170^{\circ} \mathrm{E}$ and $45^{\circ} \mathrm{S}$ ) is in the south-west corner, and the northern limit of Finland (roughly $70^{\circ}$ ) is encompassed.

The New Zealand map in Figure $2 b$ is produced with similar code.

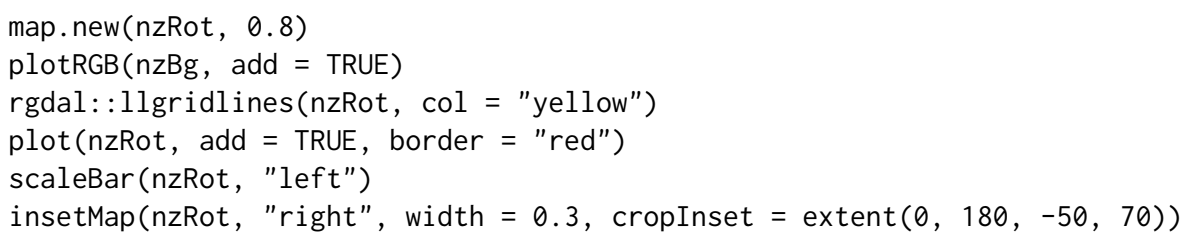

The use of plotRGB in place of plot is used for the background map, and the scale bar has been placed at the centre-left. The llgridlines function from rgdal added latitude and meridian lines in yellow.

The images in Figure 2 have dimensions of 4 by 5 inches, saved as png files with 72 pixels per inch. Executing the code above in an interactive $\mathrm{R}$ session will likely produce maps with a slightly different appearance unless the graphics window has these same dimensions. This document is produced with knitr (Xie, 2015), and the figure dimensions are set with the fig. height $=$ and fig. width $=$ options to code chunks. 
The map images in finlandBg and nzBg were retrieved from OpenStreetMap.org and MapQuest respectively, and although they are free to use and reproduce they must be attributed. The openmapAttribution function produces an attribution for an object produced by openmap or a string valid as a path $=$ argument for openmap. An attribution for nzBg (or "mapquest-sat"), as in the caption for Figure 2, is produced with

openmapAttribution $(\mathrm{nzBg}$, short $=$ TRUE $)$

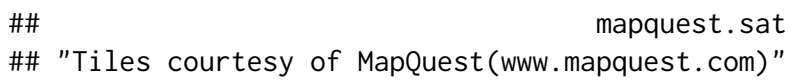

The Acknowledgements section of this paper has used this function without the short = TRUE argument.

openmapAttribution (finlandBg)

\# landscape

\#\# "copyright OpenStreetMap.org contributors. Data by OpenStreetMap.org

\# available under the Open Database License (opendatacommons.org/licenses/odbl),

\# cartography by Thunderforest.com"

The additional argument type $=$ "latex" is used in the source code for this paper, and type = "markdown" is also available.

\section{Projecting background maps}

There are a number of potential pitfalls involved when using background map images with projected data, and this section will describe some additional options to openmap and map. new which can help in this regard.

Two undesirable features of Figure $2 \mathrm{a}$ are the white triangular section in the top left of the map, and the low resolution and lack of legibility of the names of towns and cities. How this arose can be understood by contrasting the map image retrieved from OpenStreetMap.org in Figure 3a with the Finland Uniform Coordinate System map in Figure 3b. The map in Figure 3a uses coordinates in the Spherical Mercator projection, where a vertically-oriented cylinder is wrapped around a spherical Earth at the equator, ${ }^{1}$ and the rectangular area covered by the original map becomes somewhat trapezoidal when projected to the Transverse Mercator coordinates in Figure 3b. The transformation has distorted the text on the image, and the image does not completely cover the black rectangle corresponding to the plotting region of Figure $2 \mathrm{a}$.

The map images provided by OpenStreetMap.org and elsewhere are available at different zoom levels or resolutions. A map at zoom level 0 is a 256 by 256 pixel image covering the entire world. Zoom level 1 covers the world in 4 "map tiles" of 256 by 256 pixels, and zoom level $N$ consists of $4^{N}$ such tiles. The zoom level can be specified directly in openmap with the zoom $=$ argument, or indirectly with the maxTiles = argument. With the default value of maxTiles $=9$, opemnap will find the highest zoom level where the number of map tiles required to cover the spatial object supplied is at most 9. The finlandBg map has a zoom level of 5 and 6 tiles, giving a 376 by 768 pixel image. This information is contained in an attribute of finlandBg.

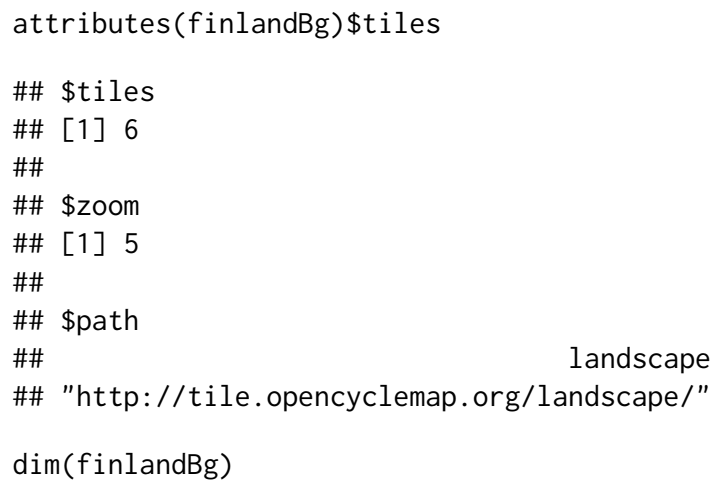

\footnotetext{
${ }^{1}$ The Web Mercator, which originated with Google Maps, is in fact slightly different from the Spherical Mercator assigned to map images by mapmisc. Maps in a Web Mercator projection are visually identical to a Spherical Mercator, but the Mercator $x-y$ coordinates are hidden and users are shown coordinates which have been converted long-lat (EPSG 4326). The group managing the EPSG codes initially refused to assign a code to the Web Mercator, reportedly saying: "We will not devalue the EPSG dataset by including such inappropriate geodesy and cartography" (Wikipedia, 2016). The Web Mercator was later assigned EPSG code 3857.
} 


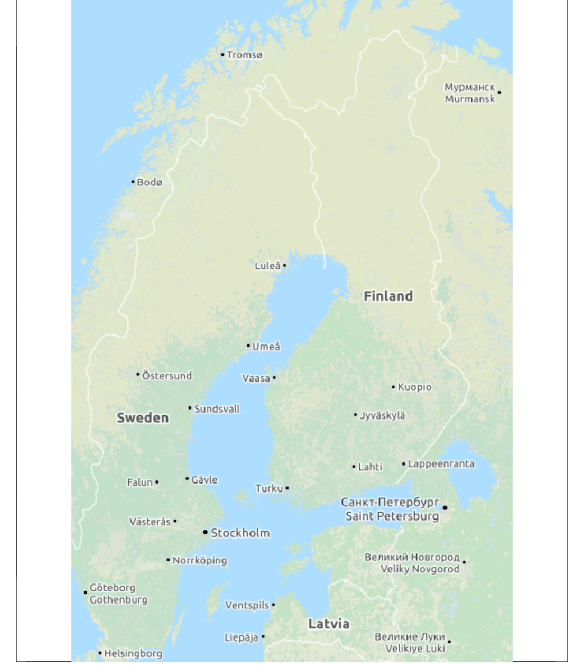

(a) Spherical Mercator

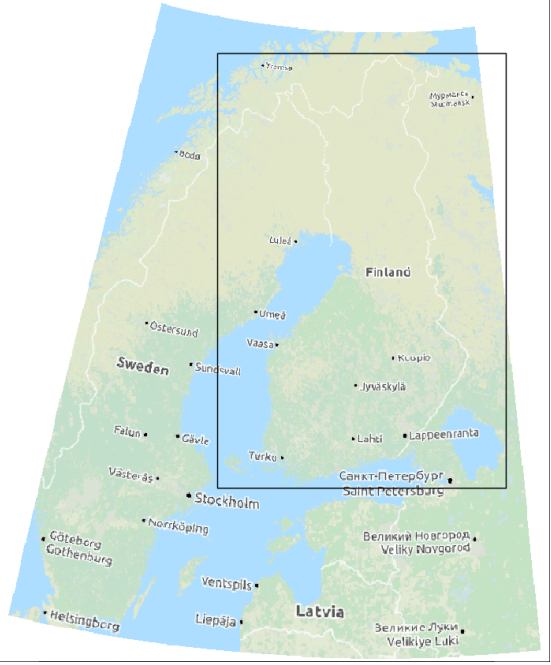

(b) Finland UCS

Figure 3: Map images of Finland in the Spherical Mercator projection and the Finland Uniform Coordinate System (UCS) projection. (C) OpenStreetMap.

\#\# [1] $\begin{array}{lll}768 & 376 & 1\end{array}$

When openmap projects the downloaded map tiles to the Finland CRS (using projectRaster from the raster package), a number of pixels from the original map are lost or put out of position and the text can become mangled.

A partial solution for improving projected images is best illustrated with the rotated CRS used for New Zealand. Figure 4a shows a map of Auckland, New Zealand, in the Spherical Mercator projection provided by OpenStreetMap.org, and the rotated Oblique Mercator projection used earlier is used for the map in Figure 4b. The map images are obtained by creating a "SpatialPoints" object for the location of Auckland in long-lat coordinates and projecting it to the CRS of the nzRot object from Figure $2 b$.

aucklandLL <- SpatialPoints(data.frame $(x=174.764204, y=-36.853744)$,

proj4string $=$ crsLL)

auckland <- spTransform(aucklandLL, projection(nzRot))

crsLL is an object in mapmisc specifying the WGS84 projection, identical to CRS ("+init=epsg: 4326). The map in Figure $4 \mathrm{~b}$ is retrieved below.

aucklandBg <- openmap (auckland, buffer $=3000$, maxTiles $=4$ )

The buffer $=$ argument specifies an additional area around auckland which the map should cover (in this case $3 \mathrm{~km}$ ), and specifying maxTiles $=4$ will select the highest zoom level which is able to cover the map region with four or fewer tiles. A map at the same zoom level in the Spherical Mercator projection, for Figure 4a, is obtained next.

aucklandBgMerc <- openmap (auckland, zoom = attributes (aucklandBg) \$tiles\$zoom, path = attributes (aucklandBg) \$tiles\$path, crs = crsMerc)

The crsMerc object gives the Spherical Mercator projection.

crsMerc

\#\# CRS arguments:

\#\# +proj=merc $+a=6378137+b=6378137+$ lat_ts $=0.0+l_{0} \_0=0.0+x_{-} 0=0.0+y_{-} \theta=0$

$\#$ \# +k=1.0 +units=m +no_defs

Figure $4 \mathrm{c}$ shows only the area within $3 \mathrm{~km}$ of the auckland object, produced by adding the buffer $=$ 3000 argument to map. new.

map. new (auckland, buffer $=3000$ )

plot (aucklandBg, add = TRUE)

scaleBar (auckland, "topleft")

One problem with Figure $4 \mathrm{~b}$ has been resolved, since the displayed area is entirely contained within the map image. The text in Figure $4 \mathrm{c}$ is visibly distorted, and the distortion is reduced by increasing 


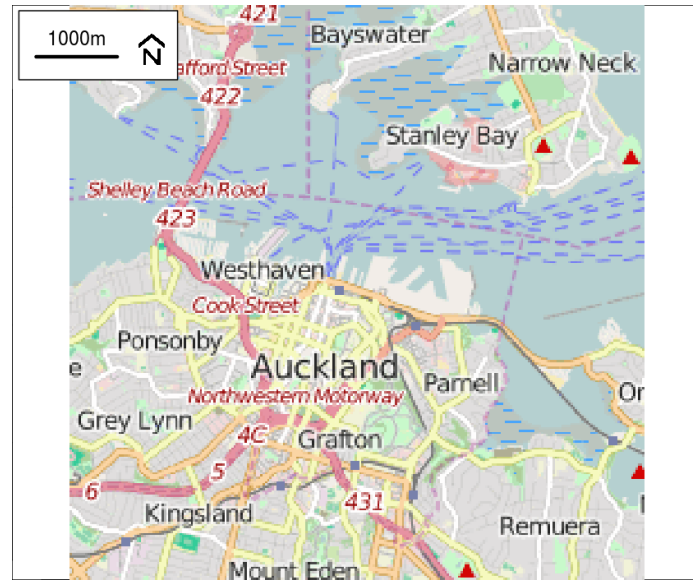

(a) Spherical Mercator

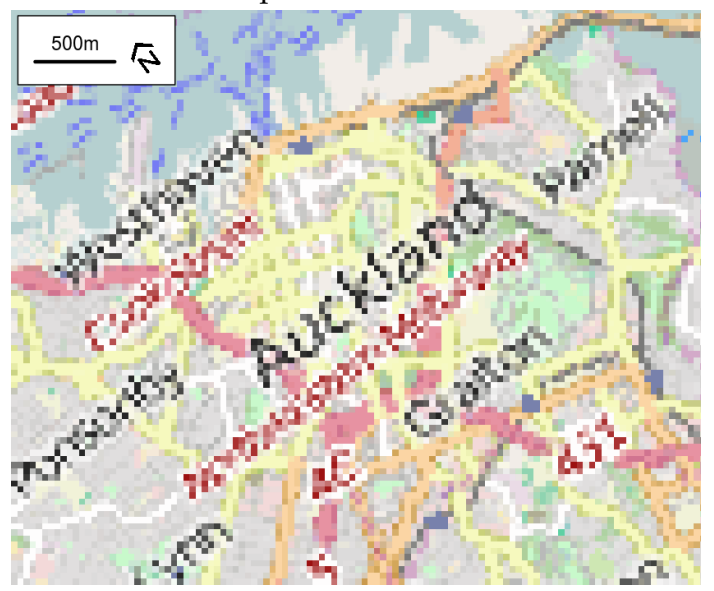

(c) Oblique, detail

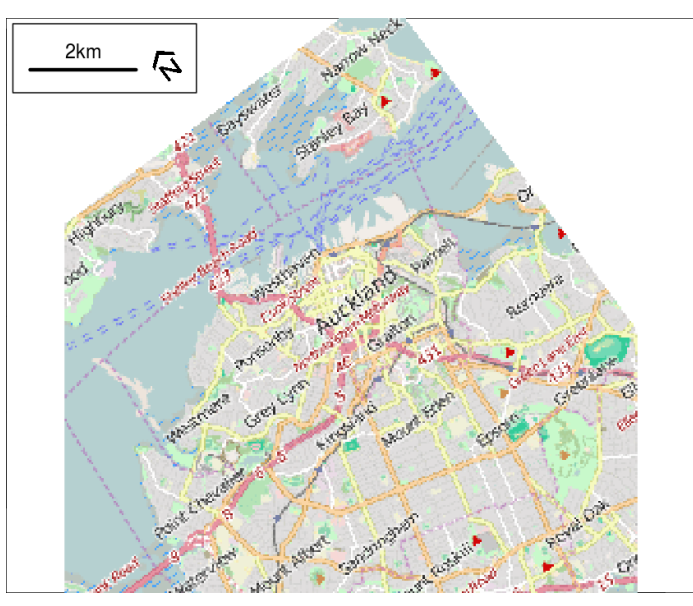

(b) Oblique Mercator

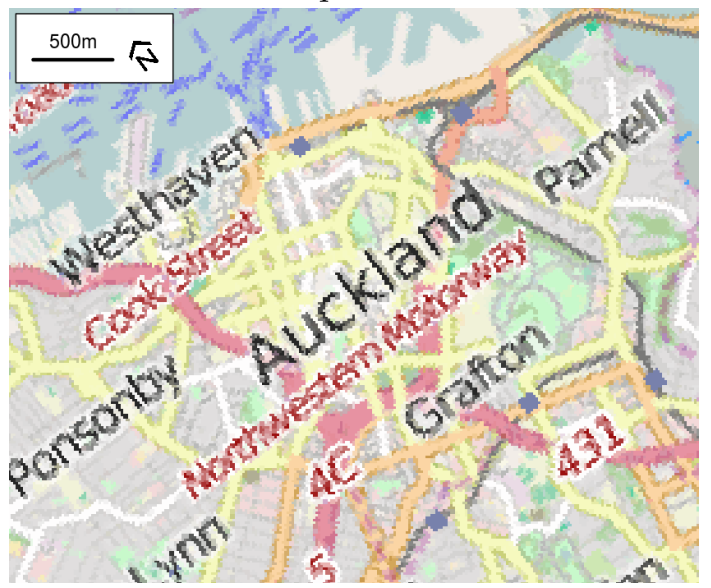

(d) Oblique, high resolution

Figure 4: Map images of Auckland, NZ, in Spherical Mercator and Oblique Mercator projections. (C) OpenStreetMap.

the resolution of the image prior to re-projection. The fact $=4$ argument to the call to openmap below increases the resolution of the raster by a factor of 4 , creating 16 times the number of pixels, yielding the map in Figure $4 \mathrm{~d}$.

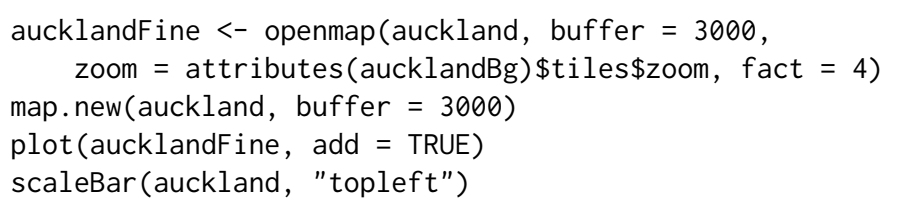

Re-projecting rasters is computationally intensive, and openmap can require considerable running time when the zoom level or fact argument are large.

\section{Equal-area map projections}

This section covers producing maps of the entire globe using the functions moll, wrapPoly, and gridlinesWrap. A number of different Coordinate Reference Systems (CRS's) are used to display maps of the world, and Munroe (2011) is a useful introduction to some of the most popular of these. Figure 5a shows the world in a Mollweide projection (Wikipedia, 2015b), an equal-area CRS with the property that the sizes of polygons on the map are roughly proportional to their true surface areas. Figures $5 \mathbf{b}, 5 \mathbf{e}$ and $5 \mathrm{f}$ use Mollweide projections with different origins and angles of orientation, projections which are obtainable with mapmisc.

Polygons corresponding to the borders of the countries of the world are contained in the wrld_simpl object from the maptools package, and this object will be used to produce the images in Figure 5 . The object is loaded with

data ("wrld_simpl", package = "maptools") 


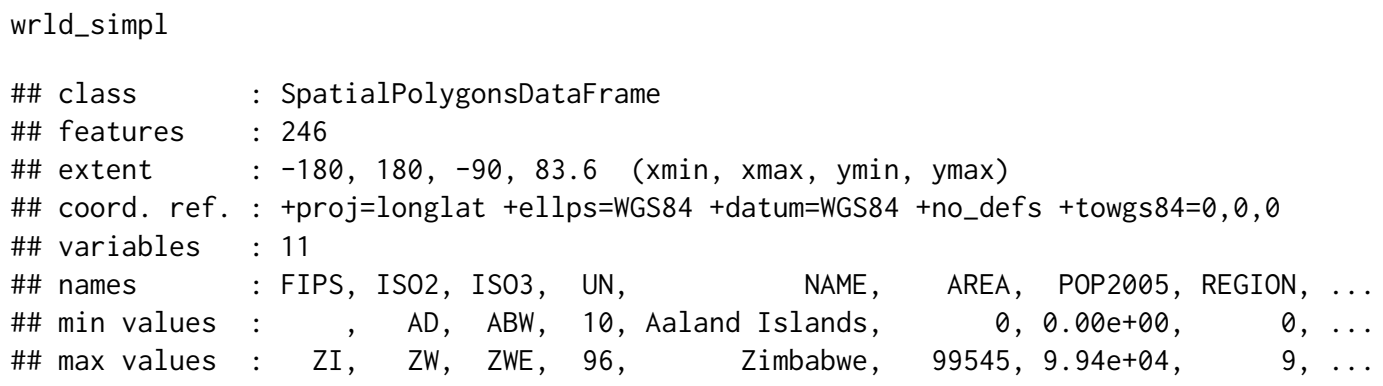

and a selection of R's named colours is assigned to the countries as follows.

colVec <- grep("gray|white|snow|ivory|turquoise|blue|[1-3]", colours(distinct = TRUE), invert $=$ TRUE, value $=$ TRUE)

wrld_simpl\$col <- rep_len(colvec, length(wrld_simpl))

The moll function creates "CRS" objects for Mollweide projections, which can be used with spTransform to compute a Mollweide projection of wrld_simpl.

mollcrs $<-\operatorname{moll}()$

worldMoll <- spTransform(wrld_simpl, mollCrs)

This "CRS" object is specified with a string similar to those seen earlier, with +proj=moll being the defining feature.

mollcrs

\#\# CRS arguments:

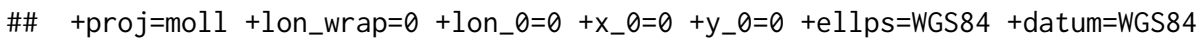

$\# \#$ +units $=m$ +no_defs +towgs $84=0,0,0$

The moll function adds two additional attributes to the "CRS" object produced, one of which is the "ellipse" object containing the spatial extent of the Earth in this projection.

names(attributes (mollCrs))

\#\# [1] "projargs" "class" "crop" "ellipse"

The map. new function uses the "ellipse" attribute to define the plotting region and add the light blue background in Figure 5 a.

map.new(mollcrs, col = "lightblue")

plot $($ worldMoll, add $=$ TRUE, col $=$ worldMoll $\$ \mathrm{col}$,

border $=\operatorname{col} 2 \mathrm{html}("$ "black", opacity $=0.2)$ )

gridlinesWrap(worldMoll, lty = 2, col = "red")

The standard Mollweide projection is symmetric about the $0^{\circ}$ Greenwich meridian line and the globe is wrapped or split in the Pacific ocean at the $180^{\circ}$ latitude line. Figure $5 \mathrm{~b}$ is centred around a meridian line passing through Hawaii and splits the Earth along a longitude line passing through Africa and Europe. The CRS for this centred Mollweide projection is produced from the moll function, which adds Hawaii's longitude to the +lon_wrap and +lon_ $\theta$ components of the CRS.

(mollHawai <- moll(geocode("hawaii")))

\#\# CRS arguments:

\#\# +proj=moll +lon_wrap $=-155.5827818+$ lon_$_{-} \theta=-155.5827818+x_{-} \theta=0+y_{-} \theta=0$

$\# \#+$ ellps $=$ WGS 84 +datum $=$ WGS 84 +units $=m+$ no_defs +towgs $84=0,0,0$

A difficulty with this Hawaiian Mollweide projection is that the meridian line along which the world is split runs through many of the polygons in wrld_simpl. The spTransform function used with this projection produces the polygons in Figure $5 c$, with horizontal lines connecting the two halves of polygons which have been split. The wrapPoly function in mapmisc addresses this problem by splitting the affected polygons prior to their projection, using the "crop" attribute of the "CRS" object produced by moll.

worldHawai i <- wrapPoly(wrld_simpl, mollHawaii) 


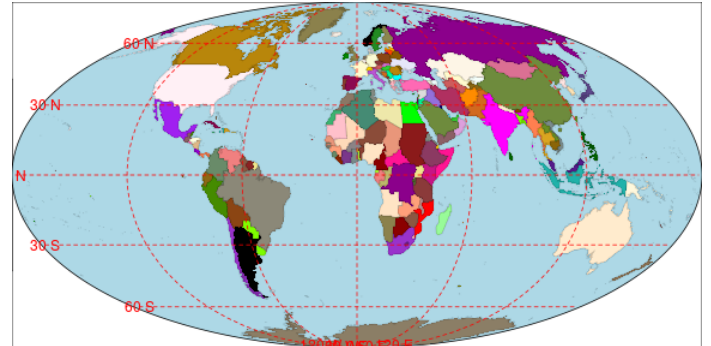

(a) Mollweide, Greenwich

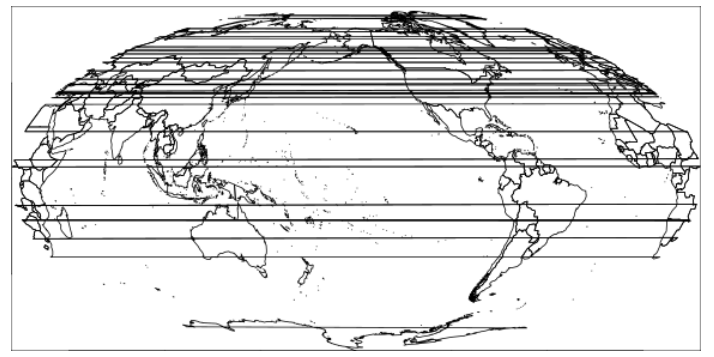

(c) Hawaii with spTransform

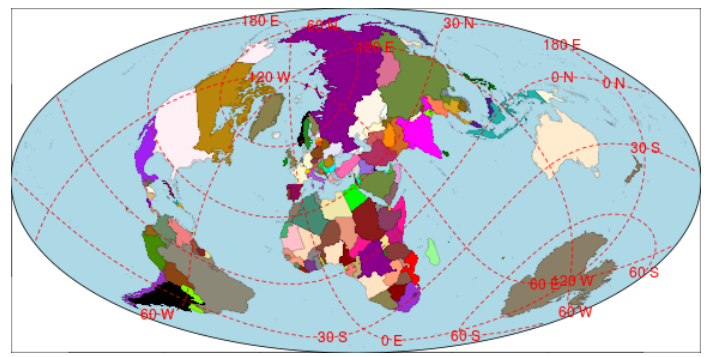

(e) Oblique Mollweide, Jerusalem

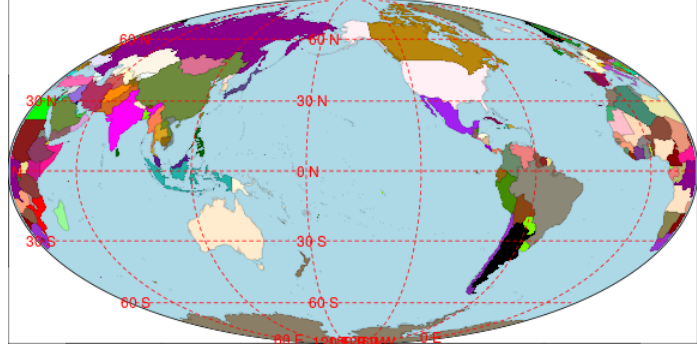

(b) Mollweide, Hawaii

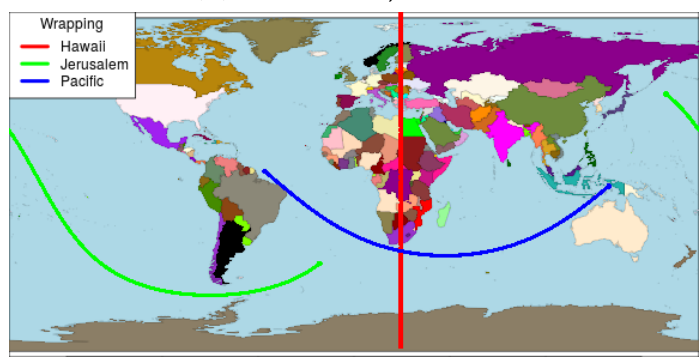

(d) Long-Lat

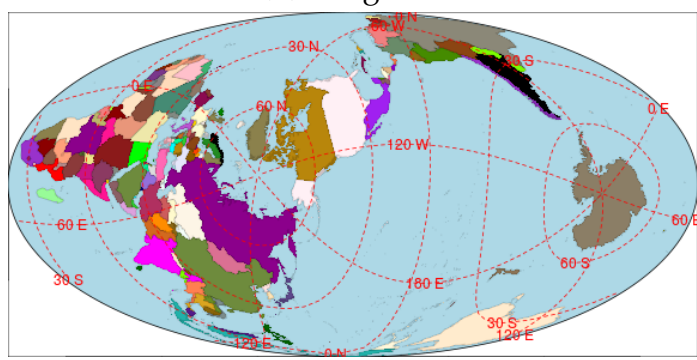

(f) Oblique Mollweide, Pacific

Figure 5: World maps in Mollweide projections.

The red vertical line in Figure $5 \mathrm{~d}$ is produced from attributes (mollHawai $\mathrm{i}$ ) $\$ c r o p$, and the function gDifference from rgeos is called by wrapPoly to remove the portion of each polygon intersecting with this line.

A Mollweide projection need not have a north-south orientation, and an Oblique Mollweide projection can be constructed by rotating the globe's long-lat coordinates to produce different origins and orientations. Figure $5 \mathrm{e}$ positions Jerusalem at the centre of the Earth (standard practice for cartographers during the middle ages), and at Jerusalem the "up" direction is $35^{\circ}$ clockwise of north.

(molloblique <- moll (geocode ("jerusalem"), angle = 35))

\#\# CRS arguments:

\#\# +proj=ob_tran +o_proj=moll +o_lon_p=-42.7134520141079

\#\# +o_lat_p=44.2305998589378 +lon_0=-17.7121046024056

\#\# +lon_wrap=-17.7121046024056 +ellps=WGS84 +datum=WGS84 +units=m +no_defs

\#\# +towgs $84=0,0,0$

This string specifies two projections are to be applied. First, +proj=ob_tran rotates the long-lat coordinates to move the north pole to the coordinate $\left(44^{\circ} \mathrm{N}, 42^{\circ} \mathrm{W}\right)$. Second, +o_proj=moll applies a Mollweide projection to these rotated coordinates using $17.7^{\circ} \mathrm{W}$ as the central meridian line. These three values $\left(44^{\circ} \mathrm{N}, 42^{\circ} \mathrm{W}, 17.7^{\circ} \mathrm{W}\right)$ are obtained by numerical optimisation, attempting to move Jerusalem as close as possible to the origin while preserving the $35^{\circ}$ orientation.

Figure $5 \mathrm{e}$ is produced by first re-projecting the world map with wrapPoly.

worldOblique <- wrapPoly(wrld_simpl, molloblique)

Adding red longitude and latitude grid lines with this projection cannot be done with the llgridlines function from rgdal, as the same horizontal striping seen in Figure $5 c$ will result. The gridlinesWrap function from mapmisc breaks the grid lines in the same manner as wrapPoly, and adds the red graticule lines as follows. 


\begin{tabular}{lrrrrrr}
\hline & Truth & Spherical Mercator & Greenwich & Hawaii & Jerusalem & Pacific \\
\hline Canada & 9.98 & 49.72 & 9.74 & 9.79 & 9.61 & 9.66 \\
Congo & 2.35 & 2.36 & 2.34 & 1.95 & 2.34 & 2.34 \\
\hline ratio & 4.26 & 21.08 & 4.17 & 5.03 & 4.11 & 4.13 \\
\hline
\end{tabular}

Table 1: Surface areas (in millions of $\mathrm{km}^{2}$ ) of Canada and Congo, and areas computed for country polygons in the Spherical Mercator projection and four Mollweide projections. Columns refer to the standard Mollweide projection (Greenwich) and the Mollweide centred on Hawaii, Jerusalem, and the north Pacific from Figures 5a, 5b, 5e and 5f.

map. new (molloblique, col = "lightblue")

plot (worldOblique, add = TRUE, col = world0blique $\$ c o l$,

border = col2html("black", 0.2))

gridlinesWrap(worldOblique, lty $=2$, col = "red")

Figure $5 \mathrm{f}$ uses an Oblique Mollweide centred in the Pacific ocean with a $85^{\circ}$ angle of rotation.

$\operatorname{crsN}<-\operatorname{moll}(c(-140,40)$, angle $=85)$

Unlike the red line of the Hawaiian projection in Figure $5 d$, the green and blue curves showing where the globe is wrapped for the two Oblique Mollweide projections lie in the ocean for the most part.

Table 1 computes the surface areas of Canada and the Congo by using the gArea function from rgeos on different transformations of the wrld_simpl polygons. The first column shows the true surface areas (according to Wikipedia) and the third row shows the ratio of Canada's surface area to the Congo's. The Spherical Mercator projection vastly over-represents the size of Canada and the four Oblique Mollweide projections are consistent in underestimating the area by a small amount. The Congo is poorly served by the Hawaiian projection, which is unsurprising as this projection splits the Congo through the middle.

\section{Custom-optimised Oblique Mercator projections}

This section describes the use of the omerc function for defining Oblique Mercator projections. Two methods of optimising map projections are implemented, with the angle of rotation chosen to produce the most compact bounding box possible or to preserve distances between a collection of points.

\section{Ad-hoc projections for compact plotting regions}

Compact representations minimising the number of void cells (i.e. those falling in the ocean) offer computational advantages when used with statistical models or software requiring data on a grid, such as the Markov random field based methods used in Brown (2015). Figure 6 shows New Zealand rotated in order to produce a compact plotting area, with bounding box having the greatest possible proportion of its area made up of land mass. This projection also minimises the number of vertical inches which Figure 6 takes up on the page, which is the reason a clockwise rotation is used in place of the anti-clockwise rotation from Figure $2 b$.

The omerc function calculates these ad-hoc projections based on the object to be re-projected and a vector of rotation angles. The first argument below is the $\mathrm{nzClip}$ object containing the cropped boundary of New Zealand, and the origin of the Oblique Mercator projection will be its centroid. A sequence of Oblique Mercator projections using Great Circles angled between 10 and 50 degrees from the north will be formulated, and the bounding box of New Zealand in each projection will be computed. The projection returned by omerc uses the angle giving the smallest such box, with the argument + alpha $=$ showing an $18.5^{\circ}$ angle in this example.

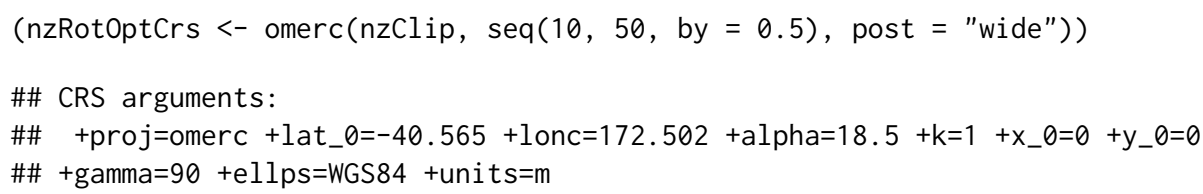

A positive angle rotates the coordinate axes clockwise, which gives the resulting map the appearance of being rotated anti-clockwise. An Oblique Mercator's Great Circle becomes the $y$-axis of the coordinate system, and a projection should seek to have as much of the area of interest as possible being close to this Great Circle. An optimal projection will therefore have a tall and narrow bounding box, in 


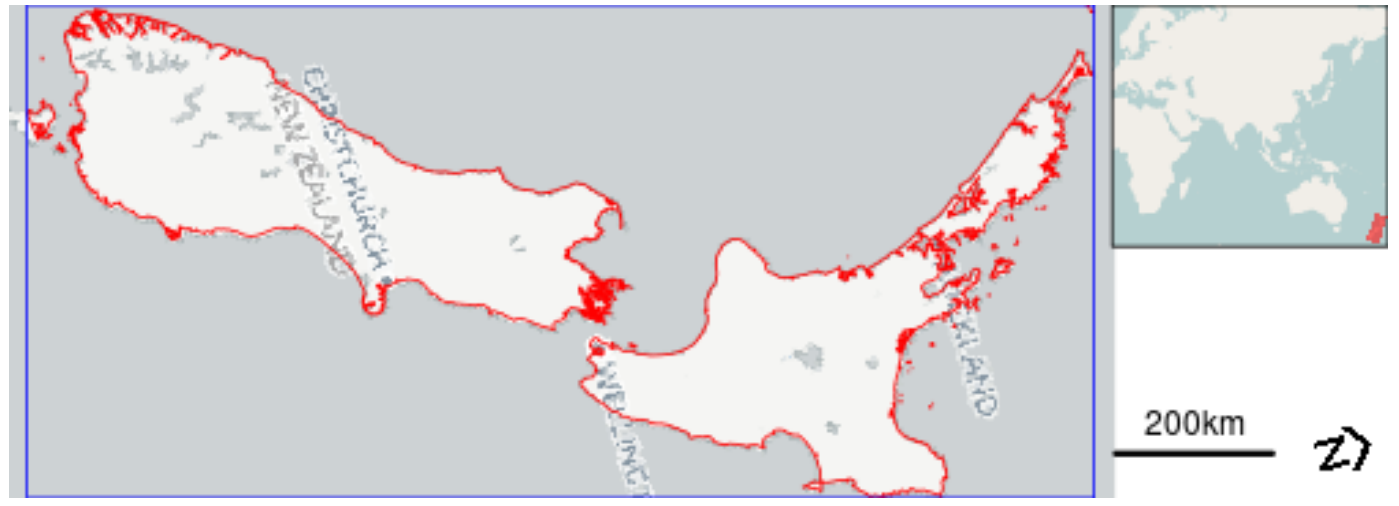

Figure 6: New Zealand in a $18.5^{\circ}$ Oblique Mercator projection ( - ) with bounding box ( - ). Background (C) CartoDB, (c) OpenStreetMap.

this instance the spine of New Zealand should run up and down the $y$-axis. The limits of 10 and 50 for the sequence of angles above are arbitrary estimates of the amount of anti-clockwise rotation required to sit New Zealand upright. The argument post $=$ "wide" in omerc specifies that a short and wide bounding box is required, and this is accomplished by rotating the planar $x-y$ coordinates post-projection using the +gamma $=90$ element.

New Zealand is re-projected and a new background map in this projection is produced below.

nzRotOpt <- spTransform(nzClip, nzRotOptCrs)

nzBgRot <- openmap (nzRotOpt, path = "cartodb", buffer = 20000, fact = 2)

Figure 6 is produced with the following.

map. new (nzRotOpt, 0.8$)$

$\operatorname{plot}(\mathrm{nzBgRot}$, add $=$ TRUE)

plot (nzRotOpt, add = TRUE, border = "red")

plot (extent (nzRotOpt), add = TRUE, col = "blue")

scaleBar (nzRotOpt, "bottomright", bty = "n")

insetMap(nzRotOpt, "topright", cropInset $=\operatorname{extent}(0,180,-50,70)$, width =0.2)

\section{Preservation of distances}

Here we seek the parameters of an Oblique Mercator projection that would preserve at best the Euclidean distances among a set of points on the surface of the Earth. This is particularly useful for large countries away from the equator and we will use Canada as an example. Figure 7 shows Canada in six different CRS's, the first of which is an Oblique Mercator optimised to preserve distances between 12 provincial and territorial capital cities. Unlike the New Zealand projection, however, the $x-y$ coordinates on the Mercator's cylinder are inverse-rotated to preserve the north-south direction as up-down.

Oblique Mercator projections are defined by an origin and an angle of rotation, only the latter is optimised by the omerc function while the origin is set by the user. Here we will set the origin somewhat arbitrarily as the town of Flin Flon, Manitoba, as an alternative to the centroid-based origin used earlier. Residents of Toronto, this author included, affectionately refer to their city as "The Centre of the Universe" 2 , but Flin Flon is a more suitable Oblique Mercator centroid for two reasons. First, Flin Flon's latitude is a useful compromise between a northerly centroid able to accommodate the Arctic islands and a centroid close to the populated areas in the south. Second, using Flin Flon as the location where the inverse rotation will preserve the north-south direction as vertical should produce a map with a familiar shape since a portion of the southern border following the $49^{\text {th }}$ parallel will be horizontal.

The locations of the cities required to compute this projection (Flin Flon and the provincial capitals) can be retrieved from Google using the dismo package. A wrapper for the geocode function in mapmisc converts the data retrieved by dismo to a "SpatialPointsDataFrame" object.

provincialCapitals <- mapmisc: :geocode(c("Vancouver, BC", "Edmonton, AB", "Regina",

"Winnipeg", "Toronto, ON", "Quebec city", "Fredericton", "Charlottetown",

\footnotetext{
${ }^{2}$ Many Canadians residing outside Toronto prefer the somewhat less affectionate nickname of "Hogtown", claiming Torontonians are arrogant and collectively self-centred.
} 
"Halifax, NS", "St Johns, NL", "Iqaluit", "Whitehorse", "Yellowknife"),

verbose $=$ TRUE)

flinflon <- mapmisc: :geocode("Flin Flon")p

The names of the provincial capitals and the location of Flin Flon are below.

provincialCapitals\$name

\begin{tabular}{|c|c|c|c|}
\hline \#\# [1] "Vancouver" & "Edmonton" & "Regina" & "Winnipeg" \\
\hline \#\# [5] "Toronto" & "Quebec City" & "Fredericton" & "Charlottetown" \\
\hline \#\# [9] "Halifax" & "St John's" & "Iqaluit" & "Whitehorse" \\
\hline \#\# [13] "Yellowknife" & & & \\
\hline ordinates(flinflon) & & & \\
\hline longitude latitude & & & \\
\hline $\begin{array}{ll}-102 & 54.8\end{array}$ & & & \\
\hline
\end{tabular}

The arguments given in the call to omerc below consist of: $x$ giving flinfon as the origin of the projection; angle giving a sequence of rotation angles to be considered; post = "north" specifying that the planar coordinates should be inverse-rotated to preserve the north direction; and preserve supplying the locations of the provincial capitals other than Iqaluit (the northerly capital of Nunavut) for calculating the distances which the projections will seek to preserve.

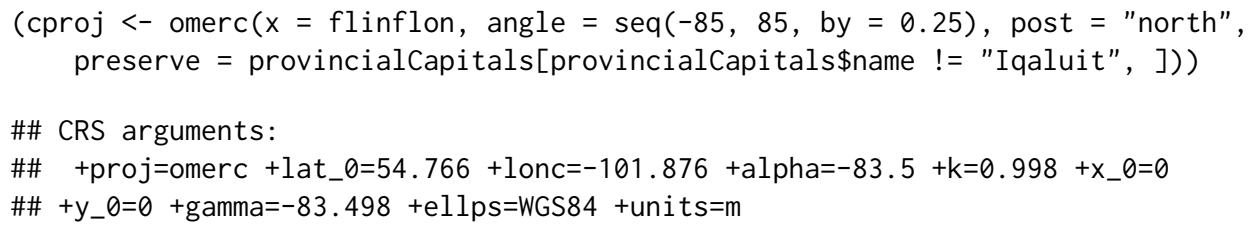

The projection above uses an origin (specified by lat_ 0 and longc) at the coordinates of Flin Flon and a cylinder tangent to the Earth along a Great Circle angled $83.5^{\circ}$ anticlockwise (given by alpha). The $\mathrm{k}$ $=0.998$ component of the CRS scales the coordinates down by a small amount $(0.2 \%)$. Although pairs of points along the Great Circle will have Euclidean distances and Great Circle distances being equal, the provincial capitals are some distance from this Great Circle and without scaling their Euclidean distances would overestimate true distances by $0.2 \%$. The gamma $=-83.5$ component appears as a consequence of having used the post $=$ "north" option, rotating the coordinates on the cylinder so that a vertical line passing through the origin contains points which are directly north or south of each other.

Two cities on Figure 7 for whom coordinates have not yet been retrieved are Kitimat, British Columbia, and the hamlet of Grise Fiord, Canada's most northerly civilian settlement ${ }^{3}$. These locations are obtained below.

moreCities <- mapmisc: :geocode(c("Grise Fiord", "Kitimat"))

cities <- bind(provincialCapitals, moreCities, flinflon)

Also shown is the Great Circle which forms the $y$-axis of the Oblique Mercator. This circle is created using one of the many useful functions in the geosphere package.

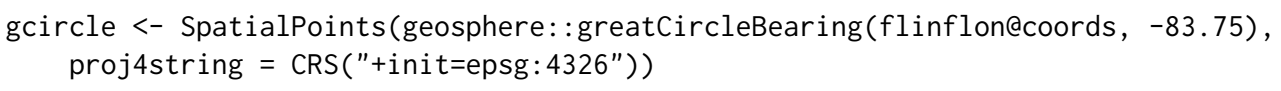

Five projections in addition to the Oblique Mercator are shown in Figure 7: a Lambert Conformal Conic projection used by the Atlas of Canada (EPSG code 3347); a Two-Point Equidistant projection based on the cities of Edmonton and Toronto (obtained from tpeqd); a Mollweide projection centred on Flin Flon; the Spherical Mercator used by OpenStreetMap.org and other internet sites; and longitudelatitude or angular coordinates. A list is created containing these four projections using mapmisc's tpeqd function in part.

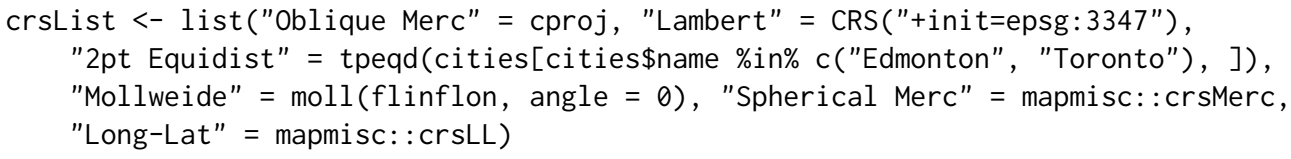

The cities and Great Circle are transformed to each of the projections with mapply.

\footnotetext{
${ }^{3}$ Readers should be aware that Grise Fiord resulted from a government-run resettlement program in the 1950's, and involved the deception and neglect of the indigenous people who were relocated to the hamlet.
} 


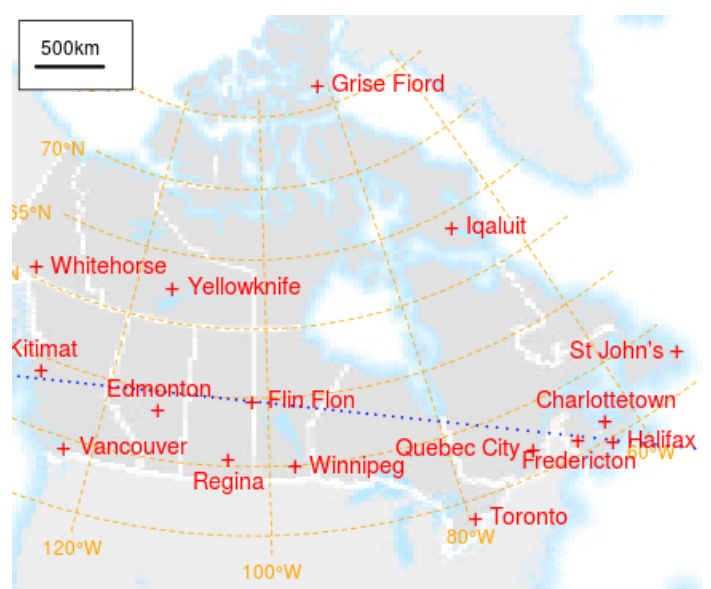

(a) Oblique Merc

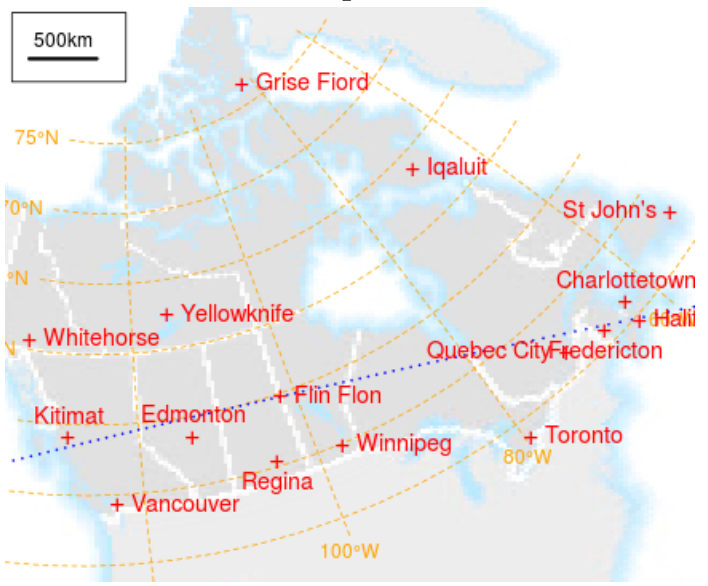

(c) 2 pt Equidist

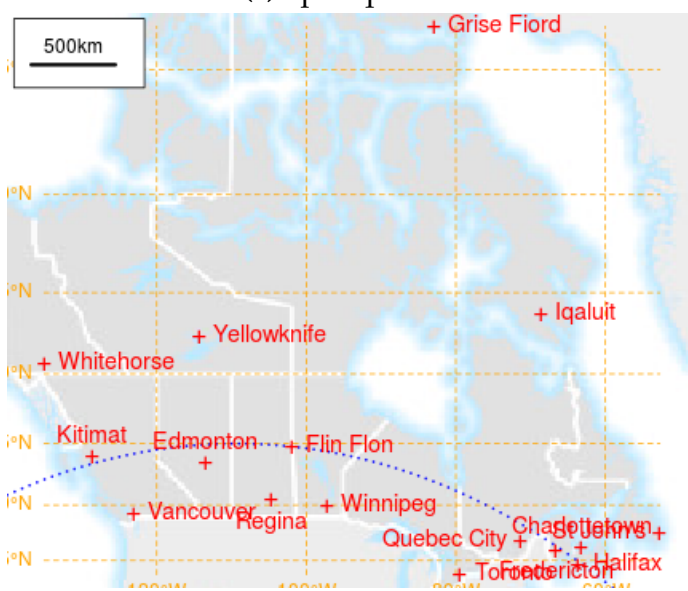

(e) Spherical Merc

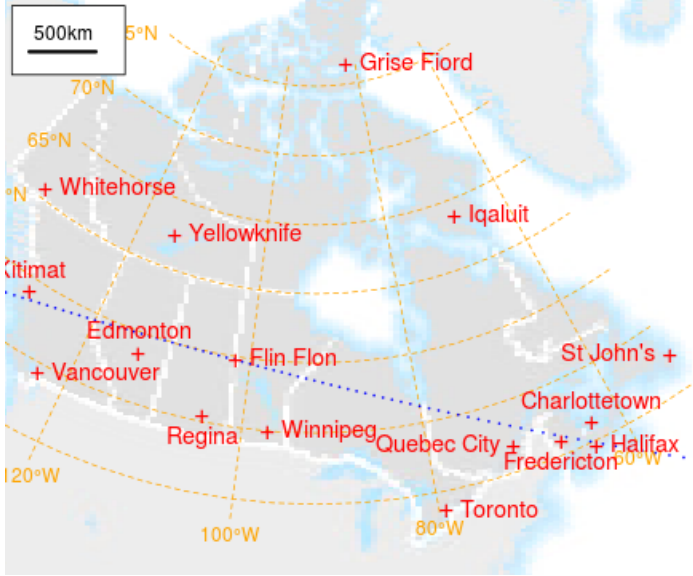

(b) Lambert

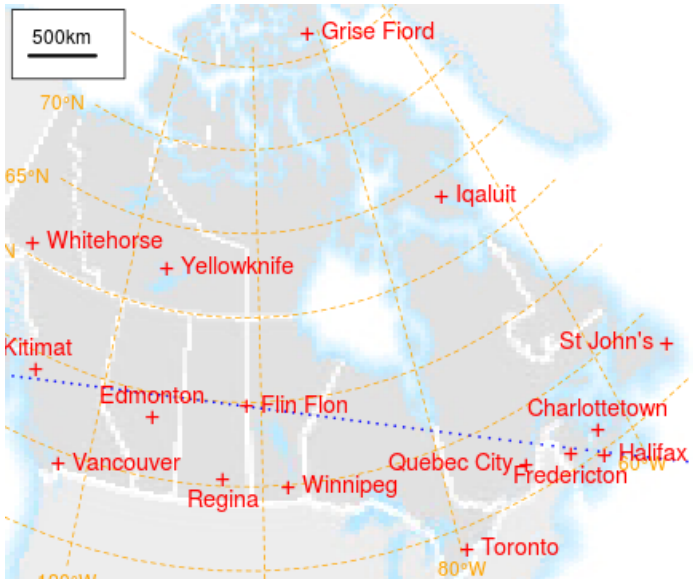

(d) Mollweide

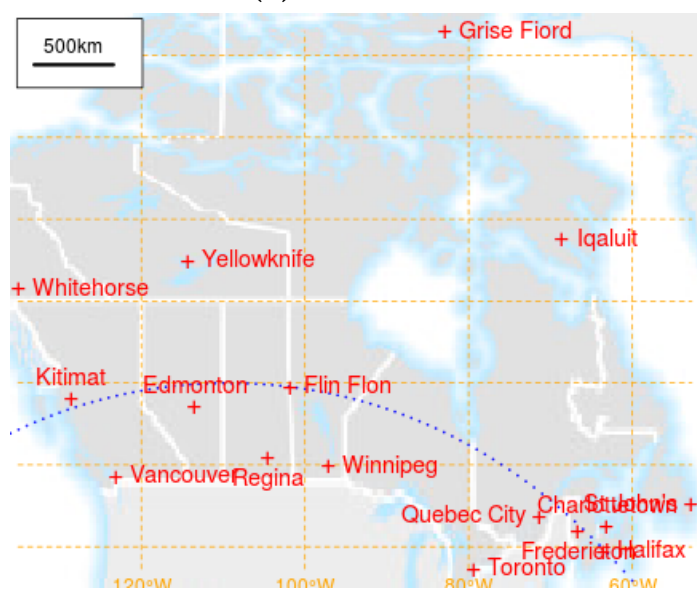

(f) Long-Lat

Figure 7: Canada and selected cities in five map projections, along with the Great Circle defining the Oblique Mercator projection ( . . ). Background from Natural Resources Canada.

citiesT <- mapply (spTransform, CRSobj = crsList, MoreArgs = list $(x=$ cities))

Code for retrieving background maps and for producing Figure 7 appears in the Appendix.

Notable features in Figure 7 are the Spherical Mercator's overemphasis of the high Arctic regions, the downward slope of the Lambert projection, the upward slope of the Two-Point Equidistant map, and the "skinny" appearance of the Mollweide. The Oblique Mercator's Great Circle, passing through Flin Flon, Halifax and Kitimat at a nearly horizontal $-83.5^{\circ}$, is necessarily a straight line in the Oblique Mercator projection and is reasonably straight in three other projections. The yellow longitude-latitude lines and three north arrows on each plot show that only the Spherical Mercator 


\begin{tabular}{crrrrr}
\hline & Oblique Merc & Lambert & 2pt Equidist & Mollweide & Spherical Merc \\
\hline south & & & & & \\
mean & -0.05 & -0.23 & -0.13 & -6.68 & 54.19 \\
sd & 0.13 & 1.03 & 0.34 & 5.22 & 8.01 \\
\hline north & & & & & \\
mean & 1.74 & -2.54 & 0.54 & -1.10 & 131.43 \\
sd & 1.65 & 0.32 & 0.81 & 6.58 & 43.82 \\
\hline
\end{tabular}

Table 2: Mean and standard deviation of the percentage by which Euclidean distance overestimates Great Circle distance for various map projections. The bottom two rows involve distances involving at least one of Grise Fiord, Iqaluit, Whitehorse and Yellowknife, with the top two rows including only distances not involving any of these four northern locations.

and long-lat projections have the property that north is in the vertical direction throughout the map.

Table 2 compares Euclidean distance to true (or Great Circle) distance for each of the map projections, with the percentage by which the former overestimates the latter for each pair of cities summarised. The first two rows give the mean and standard deviation for the percentage of overestimation of distances between the southern locations (which exclude Grise Fiord, Iqaluit, Whitehorse, and Yellowknife). The Oblique Mercator's underestimation by $0.05 \%$ betters the other projections, and the Mollweide's $6.7 \%$ underestimation is excusable given the projection's aim of preserving areas rather than distances. The Lambert's $0.23 \%$ underestimation appears respectable though the comparatively large standard deviation of $1.03 \%$ indicates several city pairs with Euclidean distances deviating several percent from their true separations. Distances involving at least one of the four northern locations are less accurate for all projections (the Mollweide notwithstanding), and the Oblique Mercator's 1.7\% overestimation betrays the fact that these northern points were not part of omerc's optimisation criteria. The Two-Point Equidistant projection is notable in its consistency and accuracy, and the Lambert projection does not appear to be a CRS which statisticians should consider using for points in Canada when accuracy of Euclidean distances is the primary concern.

\section{Maps with colour scales}

A separate set of facilities in mapmisc, complementing but disjoint from the tools related to map projections, assists with the use of colour scales and legends for maps in R. The RColorBrewer package gives $\mathrm{R}$ users access to the popular ColorBrewer collection of palettes, all of which are displayed in Table 4 in the Appendix. These colours can be used with the functions in the classInt package to create colour scales, and the venerable legend function is extremely versatile in its ability to create map legends. The steps involved in defining and using colour scales have been streamlined and consolidated into the colourScale, legendBreaks and legendTable functions in mapmisc. The process of creating suitable bins, assigning colours to data points based on these bins, specifying transparency when overlaying colours on background maps, and displaying the intervals and colours in a legend has been simplified as much as possible in mapmisc, although at least five separate lines of $R$ code are required to produce a single map.

\section{Colours with polygon data}

Consider, as a motivating example, the task of visualising the spatial variation in fertility rates in Europe using data available from the statistical office of the European Union (EUROSTAT). EUROSTAT divides the territory of the European Union and several adjoining nations into statistical units organised in a hierarchical system named "nomenclature des unités territoriales statistiques" and given the memorable acronym of NUTS. Social and demographic data on the statistical units are available at http://ec. europa. eu/eurostat/data/database, and their boundaries can be obtained from http://ec. europa. eu/eurostat/web/gisco/geodata/reference-data/administrative-units-statistical-units. Figure 8 shows the fertility rate in 2010 for 456 units of level 2 in NUTS.

Code in the Appendix for downloading and merging the boundary files and fertility rates produces a "SpatialPolygonsDataFrame" object euroF. Each polygon in the object is a territorial unit, and fertility rates for each unit are provided for the years 2001 to 2012 inclusive.

euroF

\#\# class

: SpatialPolygonsDataFrame 


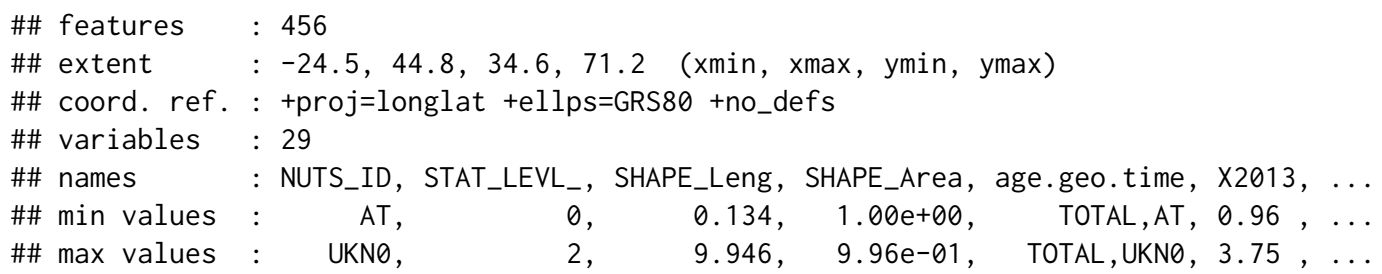

The polygons are transformed to the European Terrestrial Reference System (EPSG code 3034) below.

euroF <- spTransform(euroF, CRS("+init=epsg:3034"))

Colours in Figure 8 relate to the X2010 variable in euroF (fertility in 2010), with the colours themselves taken from the "Spectral" palette of RColorBrewer. Below the colourScale function creates eight bins (breaks = 8) for values of euroF $\$ \times 2010$, reversing the colours so that blues correspond to low values and reds are large values ( $\mathrm{rev}=\mathrm{TRUE})$.

ecol <- colourScale(euroF $\$ 2010$, col = "Spectral", breaks $=8$, rev $=$ TRUE,

style $=$ "jenks", dec $=1$, opacity $=0.5$ )

The style argument controls how the breaks are computed, and the "jenks" option corresponds to the "natural breaks" algorithm from Jenks and Caspall (1971) (see also Pebesma and Bivand, 2005, Section 3.5.2) implemented in the classIntervals function of the classInt package. A number of clustering algorithms for defining break points are provided by classIntervals, and the options for the style = argument described in the help files for classIntervals are all available using the identically named argument in colourScale. The break points are rounded to one decimal place as a consequence of the dec $=1$ argument, and the opacity $=0.5$ option gives the plotted colours $50 \%$ transparency.

The result of a call to colourScale is a list containing the break points for bins (breaks), colours associated with the bins (col), and a vector of length 456 (plot) with one colour per region.

names (ecol)

\#\# [1] "col" "breaks" "colopacity" "plot"

ecol\$breaks

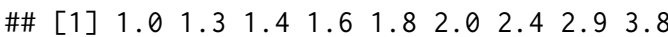

ecol\$col

\#\# [1] "\#3288BD" "\#66C2A5" "\#ABDDA4" "\#E6F598" "\#FEE08B" "\#FDAE61" "\#F46D43"

\#\# [8] "\#D53E4F"

length(ecol\$plot)

\#\#[1] 456

The breaks and col elements are used for displaying a legend, whereas the plot element can be passed as a col = argument when running plot on a "SpatialPoints" or "SpatialPolygons" object. Following the retrieval of the background map with

euroMap <- openmap (euroF, path = "osm-no-labels")

Figure 8, minus the country names, can be produced as follows.

map.new(euroF)

plot (euroMap, add = TRUE)

plot (euroF, col = ecol\$plot, add = TRUE, border = "lightgrey")

legendBreaks ("topright", ecol, title = "fertility", bg = "white")

scaleBar (euroF, "bottom", bty = "n")

Notice the $\mathrm{col}=$ ecol\$plot argument specifying the colours with which each region is filled, and that the borders of each region were given by border = "lightgrey". The legend on the right is added with legendBreaks, which passes most of its arguments to the standard legend function from the graphics package. The role of legendBreaks is to ensure the 9 numeric break points are printed near the boundaries between the coloured squares (as opposed to aligned with their centres).

The country names in Figure 8 are taken from the wrld_simpl object used earlier, although all countries smaller than Albania have been removed in order to keep the map from becoming too crowded. Below a portion of the globe in the northern hemisphere is cropped from wrld_simpl and subsequently re-projected to the European CRS. 


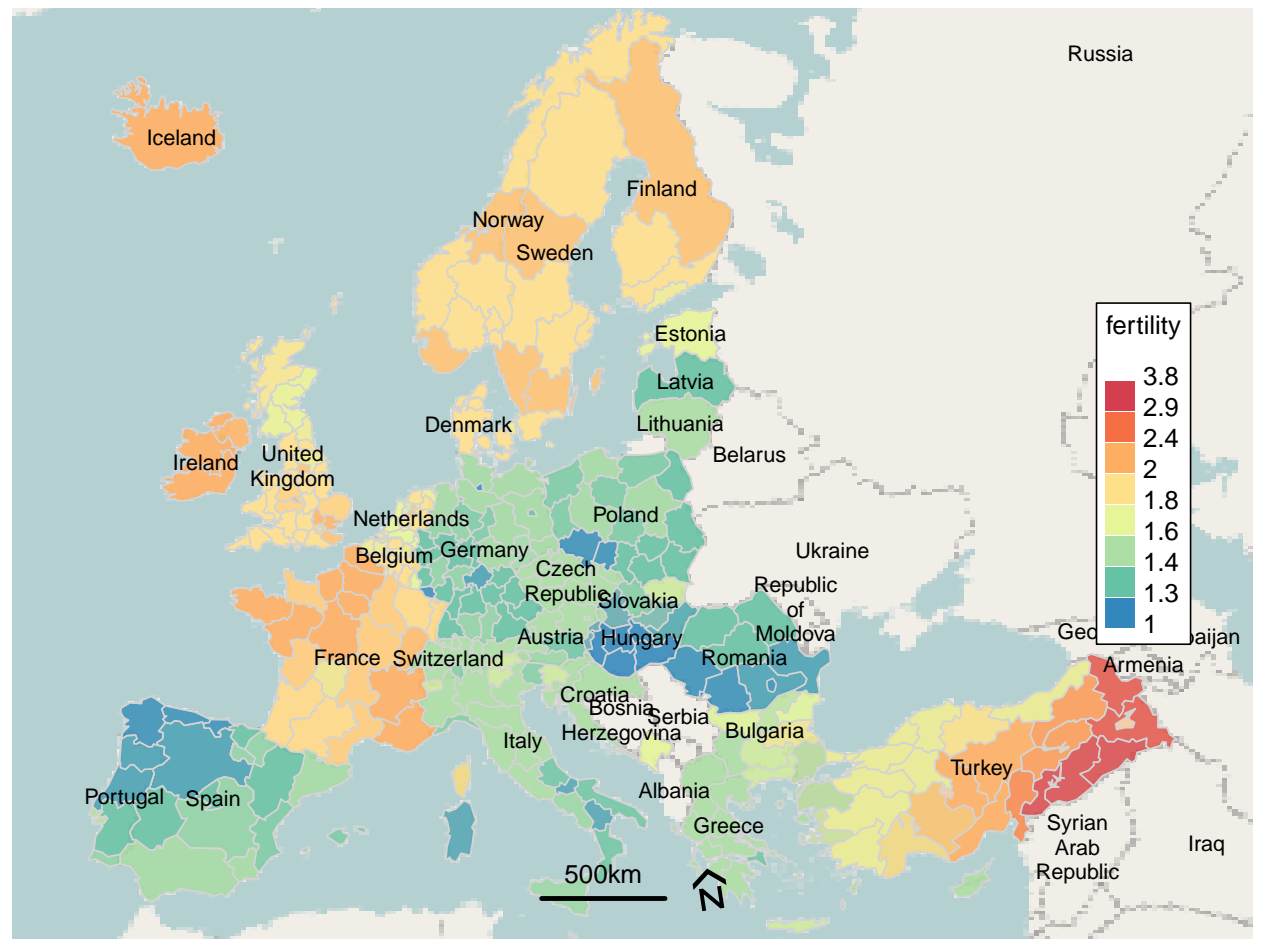

Figure 8: Fertility in Europe by NUTS. Background (C) OpenStreetMap, (C) EuroGeographics for the administrative boundaries.

data ("wrld_simpl", package = "maptools")

worldCrop <- raster: : $\operatorname{crop}\left(w r l d \_s i m p l, \operatorname{extent}(-20,100,0,90)\right.$ )

worldE <- spTransform(worldCrop, projection(euroF))

The areas of the countries are computed from the map worldMoll in the Mollweide projection, merged into the worldE object, and all countries at least as large as Albania are retained.

worldE\$area <- rgeos: :gArea(worldMoll, byid = TRUE)[as.character (worldE\$ISO3)] worldE <- worldE[worldE\$area >= worldE@data[worldE\$NAME == "Albania", "area"], ]

The names are added to the plot with the text function.

text $($ worldE, labels $=$ worldE\$NAME, cex $=0.8)$

Using the style = "jenks" argument for colourScale triggers a clustering algorithm in function classIntervals which can be time consuming for large datasets. The "quantile" and "equal" options for the style argument use quantiles and equally spaced break points respectively, with the latter able to have breaks equally spaced on a transformed scale (i.e. log or square root). Some examples appear below.

colourScale (euroF\$2010, breaks = 8, style = "quantile", dec = 1) \$breaks

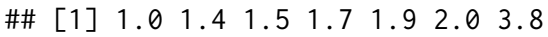

colourScale (euroF\$X2010, breaks = 8, style = "equal") $\$$ breaks

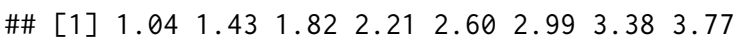

colourScale (euroF $\$ 2010$, breaks $=8$, style $=$ "equal", transform $=" l o g ") \$$ breaks

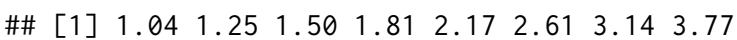

colourScale (euroF $\$ 2010$, breaks $=8$, style $=$ "equal", dec $=0) \$$ breaks

\#\# [1] $1123 \quad 3 \quad 4$

The final set of break points has a dec $=0$ argument, and although 8 breaks have been requested only 4 unique breaks remain after rounding to the nearest integer. 


\section{Rasters and colour scales}

Figure 9 shows two elevation maps of New Zealand produced with the assistance of the colourScale and legendBreaks functions. The raster package provides versions of the plot function for use with rasters, and using colourScale with rasters is slightly different from its use in the previous section.

The elevation data is obtained using the raster package's getData function.

nzAltFull <- raster: :getData("alt", country = "NZL", keepzip = TRUE)

The nzAlt object is a list of two elements, with elevation in two disjoint areas which comprise New Zealand. The first element includes the main island, its CRS is incomplete (at the time of writing) and should be re-specified.

nzAlt <- nzAltFull[[1]]

projection (nzAlt) <- CRS("+init=epsg:4326")

This raster includes a number of outlying islands, and its size becomes more manageable after the main island is extracted using the previously computed $\mathrm{nzClip}$ object.

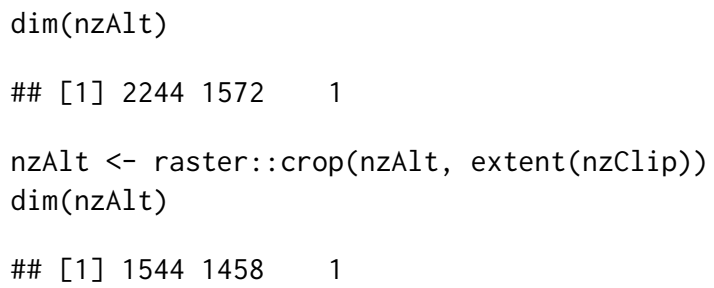

This smaller raster is now re-projected to the rotated Oblique Mercator projection used earlier, with the filename $=$ argument allowing for the resulting data to be stored as a file in R's working directory rather than in memory.

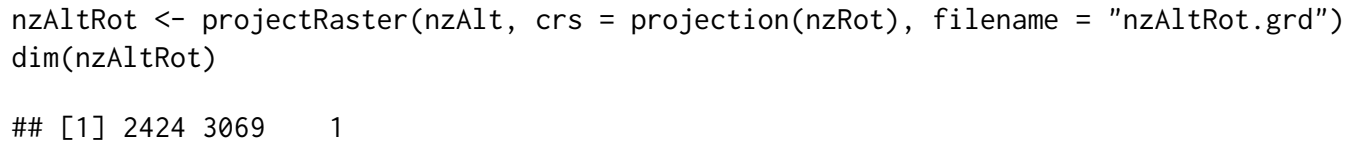

Re-projecting the raster has made it larger, as the rectangular bounding box of nzAlt becomes a diamond when rotated and nzAltRot has a bounding box large enough to contain this diamond. The crop function is used to pare this raster down to the same extent as the nzRot object.

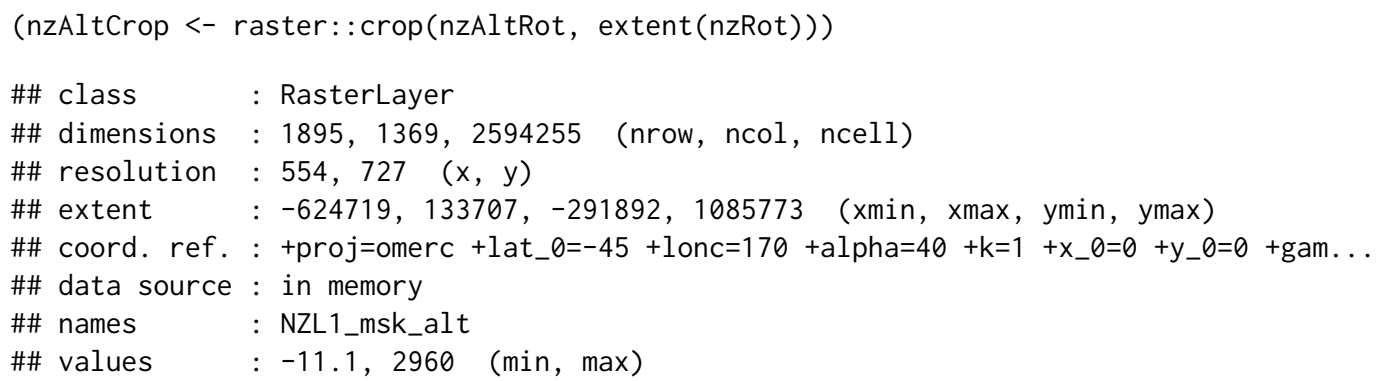

Raster images are typically large, with nzAltCrop having over 2 million cells, and computing break points using all of the data points would be time consuming. The raster package provide easy access to the maximum and minimum values ( -11 and 2960 above), which makes equally spaced break points (style = "equal" below) quick to compute. All other styles of breaks are computed using a sample of 20,000 cells taken using raster's sampleRegular function.

nzAltCol <- colourScale(nzAltCrop, breaks = 7, col = terrain.colors, style = "equal", $\operatorname{dec}=-2)$

Here the $\mathrm{col}=$ argument was given the terrain. colors function, and any function accepting a single integer argument and returning a vector with the specified number of colours would suffice. Below a second colour scale is computed using the "OrRd" colour palette and a supplied vector of breaks.

nzAltTrans <- colourScale(nzAltCrop, breaks $=c(-20,100,500,1200,2000,3100)$, col $=$ "OrRd", style = "fixed", opacity $=c(0.2,1))$ 


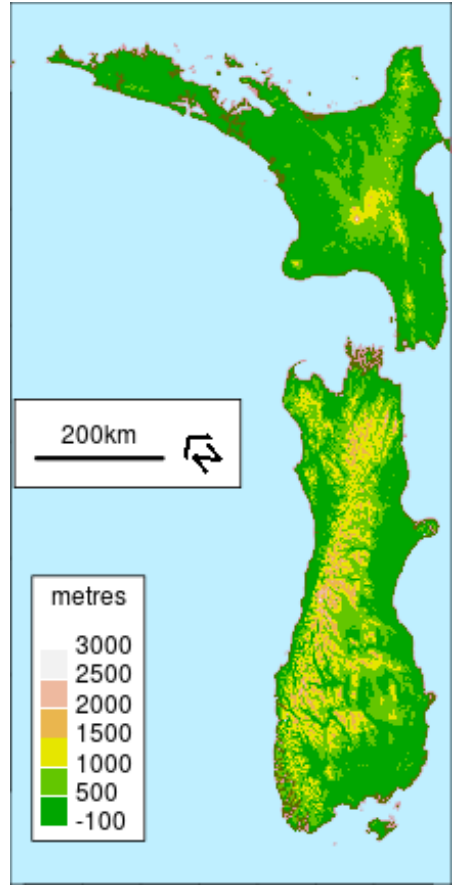

(a) terrain.colors()

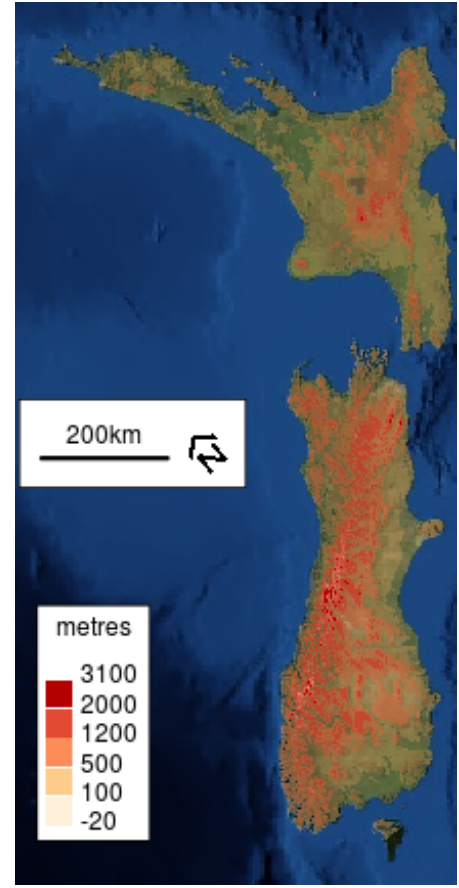

(b) "OrRd" colours with opacity. Tiles courtesy of MapQuest.

Figure 9: Elevation maps of New Zealand using colours from terrain. colors and the "OrRd" colours from RColorBrewer.

Here the opacity argument has been given a vector of length 2, giving the opacity of the first colour and last colour respectively with intervening colours having opacities which are linear interpolations of these values.

Figure $9 \mathrm{a}$ is produced with code below.

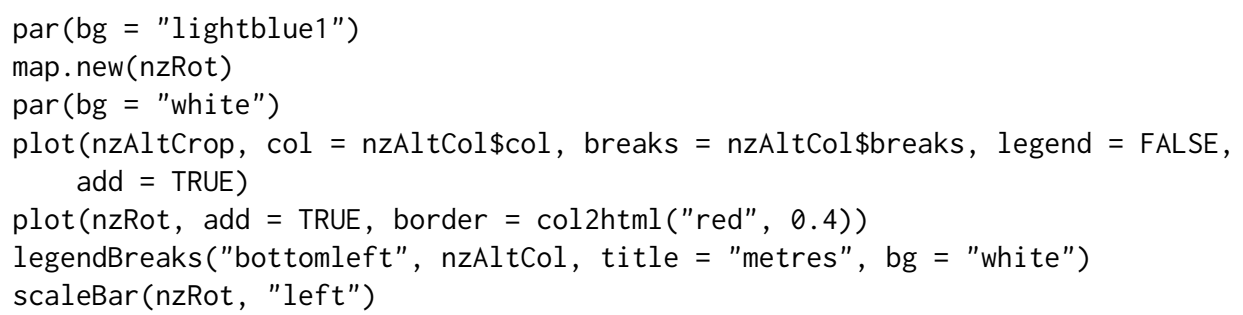

No background map is present, though the background has been set to a sea-like colour. The line of code beginning with plot (nzAltRot, ... adds the elevation data to the map. The col and breaks elements of the colour scale nzAltCol are passed as identically named arguments of the plot method from the raster package. The legend = FALSE argument prevents plot from adding its own legend, which would not fit well with this map as it always appears on the right.

The code for Figure $9 \mathrm{~b}$ is below.

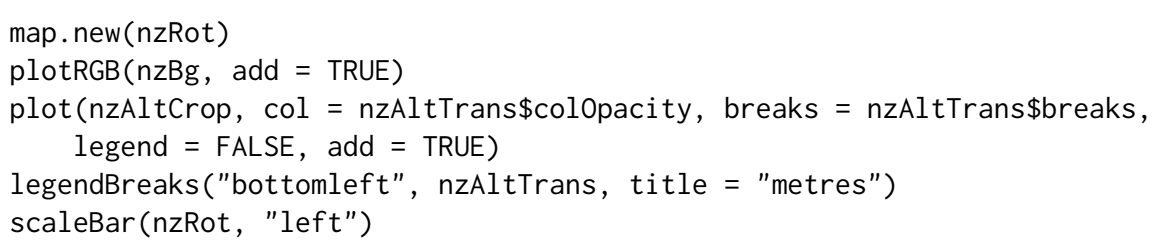

Here the colOpacity element of the colour scale, which has 2-digit opacity levels appended to each specified colour, is passed as col = argument to allow the satellite photo beneath to be viewed.

\section{Colours with categorical data}

Figure 10 maps land categories in Africa, and colourScale has been used to assign colours to 10 of the 20 land categories present. The way the raster package treats categorical data (a factor in R parlance) 
requires a slightly different use of colourScale from the previous section, and legends suitable for categorical data can be produced by legendBreaks and the related function legendTable.

The land cover data originate from the European Space Agency, and are redistributed by worldgrids.org. The file is provided in . tif format, compressed as a (.gz) file. The code below downloads, unzips, and loads the data into $\mathrm{R}$.

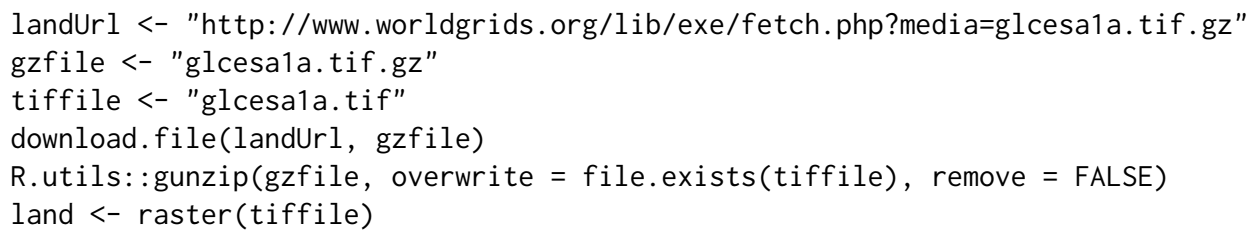

This land raster object spans the entire globe, and a portion of central Africa containing both Liberia and Tanzania is extracted with the help of the wrld_simpl object used earlier.

worldSub <- wrld_simpl[grep("Liberia|Tanzania", wrld_simpl\$NAME), ]

worldSub <- spTransform(worldSub, projection(land))

landSub <- raster: : $\operatorname{crop}($ land, extend(extent(worldSub), 5))

The extend function has added an additional 5 units (in this case degrees latitude and longitude) to the region to be extracted.

A text file containing a list of land categories and their numeric identifiers is posted at worldgrids.org, it is retrieved and loaded below.

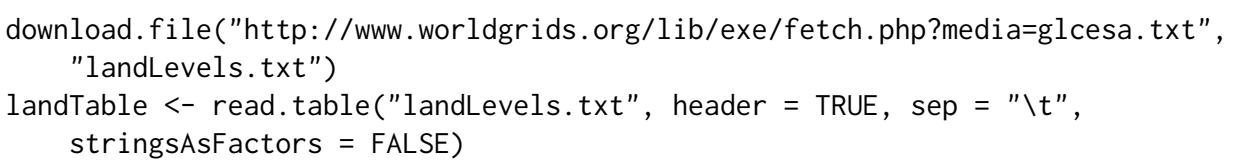

This table can be used with colourScale to produce map colours and a legend, although the table must first be modified as colourScale expects it to include a numeric ID column and a column called label of descriptions. The land categories are assigned integer values in the raster

unique (landSub)

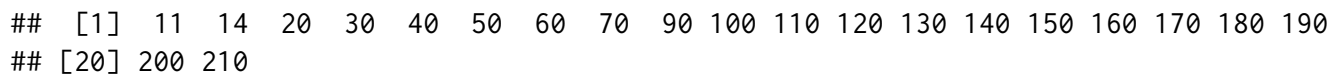

which correspond to the trailing digits of the DESCRIPTION column of the table.

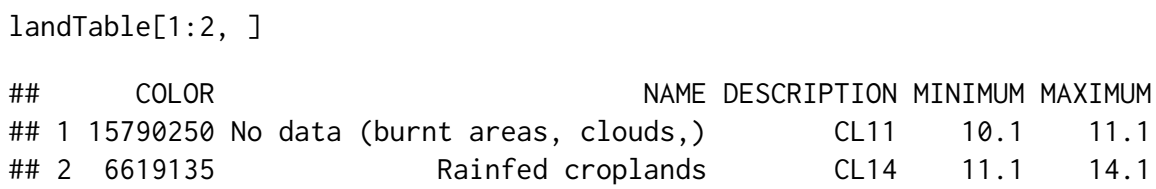

The codes are converted into the numeric ID variable and the NAME column tidied up with a complex gsub statement and saved as label below.

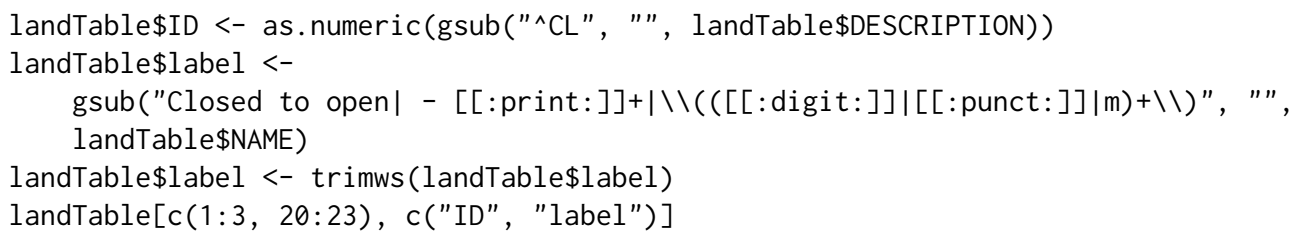

The labels argument is used to provide information on categories and labels to colourScale, and it requires a data. frame with columns named ID and label giving category identifiers and descriptions respectively. 
landLevels <- colourScale(landSub, breaks $=10$, style $=$ "unique", col = "Set3", exclude $=c(11,210,220,230)$, labels $=$ landTable $)$

Here 10 colours have been requested from the "Set 3 " palette, with style = "unique" specifying that the data are categorical rather than continuous. The 10 most common land types will be assigned colours, although the exclude $=$ argument specifies that several categories (i.e. 11 no data, 210 water bodies, 200 bare areas) will not be colour-coded regardless of how prevalent they are.

names (landLevels)

\#\# [1] "col" "breaks" "colopacity" "colourtable" "colortable"

\#\# [6] "levels" "legend"

The colourtable and levels elements above were not present when colourScale was used in the previous section, and these elements will be produced whenever a labels $=$ argument has been provided. The levels element is a data frame with one row per land category and columns with labels and colours, and is compatible with the raster package's facilities for categorical variables. A categorical raster has a list of data frames, one for each raster layer, accessible by executing levels (landSub). The land categories are the first and only layer of the landSub raster and the table produced by colourScale is added to the first element of the levels list below.

levels(landSub)[[1]] <- landLevels $\$$ levels

The colourtable object in landLevels is a vector of colours associated with each numeric category, with NA's for those categories for which no colour has been assigned (ID 201, Water Bodies for example). It will be used by raster's plotting functions if it has been added to the legend@colortable slot of a raster as follows.

landSub@legend@colortable <- landLevels\$colourtable

The American spelling "color" is used by the majority of R packages, despite the Guidelines for Rd files stating: "For consistency, aim to use British (rather than American) spelling." This author, being Canadian, requires "color" and "colour" to be interchangeable and provides landLevels $\$$ colortable (and a colorscale function) to this effect.

Figure 10 includes the "stamen-toner" web map as a foreground (rather than background) layer with country borders and names. The tonerToTrans function converts the white pixels in the "stamen-toner" map to transparent (and greys to semi-transparent), allowing the map to be added after and on top of the land category image.

landMap <- openmap (landSub, path = "stamen-toner")

landMapTrans <- tonerToTrans(landMap)

Figure 10 is produced as follows.

map.new(landSub)

plot (landSub, add $=$ TRUE)

plot (landMapTrans, add $=$ TRUE)

legendBreaks ("bottomleft", landSub, ncol $=2$, width $=25$, lines $=3$,

text.col = "yellow", cex $=0.8$, pt.cex $=3$, inset $=0$, bty $=" n "$ )

The landSub raster, having had landLevels $\$$ colourtable and landLevels $\$$ levels attached to it, is the only object required by the plot and legendBreaks functions. The legendBreaks function passes the ncol, cex and inset arguments to the legend function, arguments specifying two columns, slightly smaller than normal text, and a position flush to the bottom left respectively. The width $=25$ argument inserts line breaks in the legend labels after 25 characters, and the lines $=3$ argument causes only the first three lines to be displayed.

An alternative to including the legend on the plot is to create an "in-line" legend as in the caption to Figure 10. The legendTable function assists in this task, with the code

legendTable(landSub, collapse ="; ", type = "latex")

used in this instance. Table 3 is created with the following.

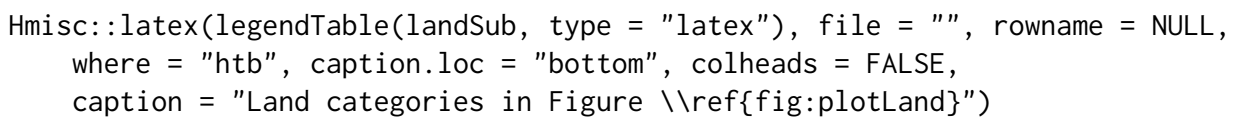




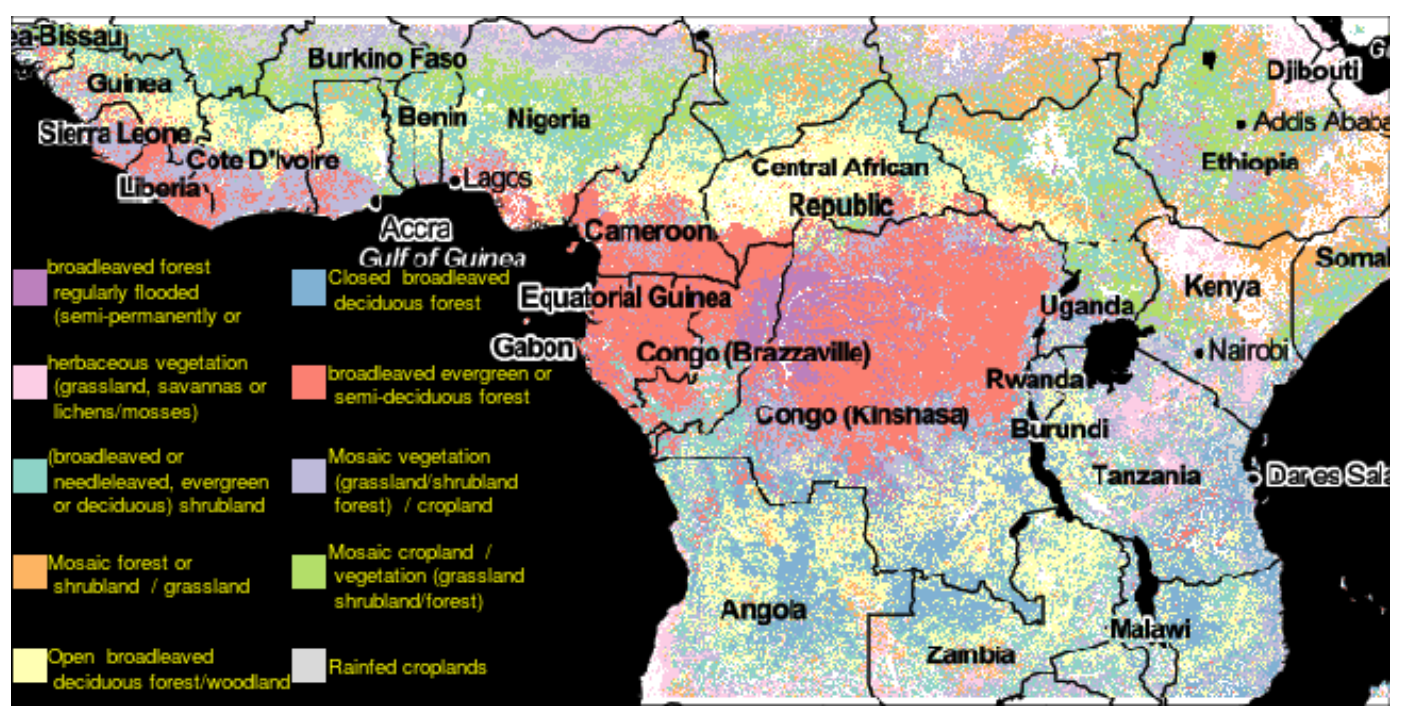

Figure 10: Land categories in central Africa: Rainfed croplands ( $\quad$ ); Mosaic cropland / vegetation (grassland/shrubland/forest) ( _ ); Mosaic vegetation (grassland/shrubland/forest) / cropland ( $\quad$ ); Broadleaved evergreen or semi-deciduous forest ( $\quad$ ); Closed broadleaved deciduous forest ( $\quad$ ); Open broadleaved deciduous forest/woodland ( $\quad$ ); Mosaic forest or shrubland / grassland ( $\square$ ); (Broadleaved or needleleaved, evergreen or deciduous) shrubland ( $\square$ ); Herbaceous vegetation (grassland, savannas or lichens/mosses) ( $\quad$ ); Broadleaved forest regularly flooded (semi-permanently or temporarily) (__ ). Background ( ) Stamen Design.

\begin{tabular}{l}
\hline Rainfed croplands \\
Mosaic cropland / vegetation (grassland/shrubland/forest) \\
Mosaic vegetation (grassland/shrubland/forest) / cropland \\
Broadleaved evergreen or semi-deciduous forest \\
Closed broadleaved deciduous forest \\
Open broadleaved deciduous forest/woodland \\
Mosaic forest or shrubland / grassland \\
(Broadleaved or needleleaved, evergreen or deciduous) shrubland \\
Herbaceous vegetation (grassland, savannas or lichens/mosses) \\
Broadleaved forest regularly flooded (semi-permanently or temporarily) \\
\hline
\end{tabular}

Table 3: Land categories in Figure 10.

\section{Conclusions}

This paper and the mapmisc package aim to contribute to and advance the suite of tools available to the growing community of $\mathrm{R}$ users performing advanced statistical analyses of spatial data. The first objective of mapmisc is removing barriers to using a map projection which is appropriate for the problem at hand, even when a non-standard projection optimised for a particular study region is most appropriate. A second objective is the provision of tools which simplify the creation and use of colour scales and legends. The way in which mapmisc seeks to accomplish these goals is by automating many of the tasks involved, with obtaining and re-projecting map tiles or creating colour scales with transparency being two examples. New functionality provided by mapmisc to R users includes the creation of optimised map projections and the creation of inset maps and scale bars for data in a rotated projection.

The scope of mapmisc has been kept deliberately narrow. Maps are static rather than interactive, base graphics are used, all spatial data types used are from sp or raster, and functions have been kept simple with few arguments. In contrast to spplot from package sp and ggplot2 (Wickham, 2009), maps made with mapmisc are produced with a sequence of independent function calls with each function performing a very specific task. This approach was originally intended to benefit students and non-specialists, and the package grew out of code originally provided to students in an undergraduate course. Many of the tools in mapmisc can, however, be used with ggplot2, leaflet (Cheng and Xie, 2016) or other advanced graphical packages. No new object classes have been created by mapmisc, any package compatible with raster can use background maps from openmap and a "CRS" object 
provided by omerc or moll can be used with any package that calls rgdal for transformations. Colours and break points from colourScale can be used with spplot, and legendBreaks can be used in any graphics environment where the legend function operates.

Additional motivations for the framework mapmisc employs are reproducibility and consistency when producing multiple maps. Colour scales and background maps are defined before a map is produced, and plot areas are laid out before any graphics are added. While sometimes clumsy for interactive use, mapmisc code fits tidily within Sweave or knitr documents and script files. Reproducibility of research results is an area where R excels, and mapmisc can help to simplify the creation of high quality maps in reproducible code scripts. For refining and manually polishing maps and plots, R will never replace Geographical Information Systems or graphics editing software. For most other tasks faced by a Spatial Statistician, however, the occasions when an environment other than $\mathrm{R}$ is required are becoming fewer in number over time.

\section{Acknowledgements}

The author holds a Discovery Grant from the Natural Sciences and Engineering Council of Canada.

\section{Attributions for background maps}

Figures 2a, 8 and all inset maps: (C) OpenStreetMap contributors. Data by OpenStreetMap available under the Open Database License, cartography is licensed as CC BY-SA.

Figure 2b, 9b: Tiles courtesy of MapQuest, portions courtesy NASA/JPL-Caltech and U.S. Depart. of Agriculture, Farm Service Agency.

Figure 7 Cartography by The Canada Base Map - Transportation (CBMT) web mapping services of the Earth Sciences Sector (ESS) at Natural Resources Canada (NRCan) licensed as the Open Government Licence - Canada.

Figure 10: Map tiles by Stamen Design under CC BY 3.0. Data by OpenStreetMap available under the CC BY-SA.

Figure 6: Map tiles by CartoDB under CC BY 3.0. Data by OpenStreetMap available under the Open Database License.

Figure 11: The Appendix is (C) OpenStreetMap contributors. Data by OpenStreetMap available under the Open Database License, cartography is licensed as CC BY-SA. with the exceptions below.

mapquest : Tiles courtesy of MapQuest. Data by OpenStreetMap available under the Open Database License.

mapquest-sat : Tiles courtesy of MapQuest, portions courtesy NASA/JPL-Caltech and U.S. Depart. of Agriculture, Farm Service Agency.

mapquest-labels : Tiles courtesy of MapQuest. Data by OpenStreetMap available under the Open Database License.

maptoolkit : ( ) Toursprung GmbH Data by OpenStreetMap available under the Open Database License.

humanitarian : (C) OpenStreetMap contributors. Data by OpenStreetMap available under the Open Database License, cartography by Humanitarian OSM team is licensed as CC BY-SA.

cartodb : Map tiles by CartoDB under CC BY 3.0. Data by OpenStreetMap available under the Open Database License.

cartodb-dark : Map tiles by CartoDB under CC BY 3.0. Data by OpenStreetMap available under the Open Database License.

stamen-toner : Map tiles by Stamen Design under CC BY 3.0. Data by OpenStreetMap available under the Open Database License.

stamen-watercolor : Map tiles by Stamen Design under CC BY 3.0. Data by OpenStreetMap available under the CC BY-SA.

\section{Bibliography}

H. Bengtsson. R.utils: Various Programming Utilities, 2016. URL https://CRAN.R-project.org/ package=R.utils. R package version 2.3.0. [p64] 
R. Bivand. classInt: Choose Univariate Class Intervals, 2015. URL https://CRAN.R-project.org/ package $=$ classInt. R package version 0.1-23. [p64]

R. Bivand and N. Lewin-Koh. maptools: Tools for Reading and Handling Spatial Objects, 2016. URL https: //CRAN. R-project.org/package=maptools. R package version 0.8-39. [p64]

R. Bivand and C. Rundel. rgeos: Interface to Geometry Engine - Open Source (GEOS), 2016. URL https://CRAN.R-project. org/package=rgeos. R package version 0.3-19. [p64]

R. Bivand, T. Keitt, and B. Rowlingson. rgdal: Bindings for the Geospatial Data Abstraction Library, 2016. URL https://CRAN. R-project.org/package=rgdal. R package version 1.1-8. [p64]

R. S. Bivand, E. Pebesma, and V. Gomez-Rubio. Applied Spatial Data Analysis with R. Springer, New York, 2nd edition, 2013. URL http://www. asdar-book. org/. [p65]

P. Brown. mapmisc: Utilities for Producing Maps, 2016. URL https://CRAN. R-project. org/package= mapmisc. R package version 1.5.0. [p64]

P. E. Brown. Model-based geostatistics the easy way. Journal of Statistical Software, 63(12):1-24, 2015. URL http://www. jstatsoft.org/v63/i12/. [p66,75]

J. Cheng and Y. Xie. leaflet: Create Interactive Web Maps with the JavaScript "Leaflet" Library, 2016. URL https://CRAN.R-project.org/package=leaflet. R package version 1.0.1. [p86]

R. J. Hijmans. geosphere: Spherical Trigonometry, 2015a. URL https://CRAN. R-project. org/package= geosphere. R package version 1.5-1. [p64]

R. J. Hijmans. raster: Geographic Data Analysis and Modeling, 2015b. URL https://CRAN. R-project. org/package=raster. R package version 2.5-2. [p64]

R. J. Hijmans, S. Phillips, J. Leathwick, and J. Elith. dismo: Species Distribution Modeling, 2016. URL https: //CRAN. R-project.org/package=dismo. R package version 1.0-15. [p64]

G. F. Jenks and F. C. Caspall. Error on choroplethic maps: Definition, measurement, reduction. The Annals of the Association of American Geographers, 61(2):217-244, 1971. [p80]

R. Munroe. Map projections. xkcd Web Comic, 2011. URL http://xkcd.com/977. [Online; accessed 11-March-2015]. [p72]

E. Neuwirth. RColorBrewer: ColorBrewer Palettes, 2014. URL https://CRAN. R-project. org/package= RColorBrewer. R package version 1.1-2. [p64]

E. J. Pebesma and R. S. Bivand. Classes and methods for spatial data in R. R News, 5(2):9-13, November 2005. URL http://CRAN.R-project.org/doc/Rnews/. [p64, 80]

J. P. Snyder. Map projections - A working manual. Professional Paper 1395, US Geologic Survey, Washington, DC, 1987. URL http://pubs.usgs.gov/pp/1395/report.pdf. [p66]

H. Wickham. ggplot2: Elegant Graphics for Data Analysis. Springer-Verlag New York, 2009. URL http://ggplot2.org. [p86]

Wikipedia. Great-circle distance - Wikipedia, The Free Encyclopedia, 2015a. URL https:// en. wikipedia.org/w/index.php?title=Great-circle_distance\&oldid=688488703. [Online; accessed 3-November-2015]. [p66]

Wikipedia. Mollweide projection - Wikipedia, The Free Encyclopedia, 2015b. URL https://en. wikipedia.org/w/index.php?title=Mollweide_projection\&oldid=689173007. [Online; accessed 25-November-2015]. [p72]

Wikipedia. Web Mercator - Wikipedia, The Free Encyclopedia, 2016. URL https://en. wikipedia. org/w/index.php?title=Web_Mercator\&oldid=714750105. [Online; accessed 19-May-2016]. [p70]

Y. Xie. Dynamic Documents with R and knitr. Chapman and Hall/CRC, Boca Raton, Florida, 2nd edition, 2015. URL http://yihui.name/knitr/. [p69]

Patrick Brown

Cancer Care Ontario

620 University Ave

Toronto, ON M5G 2L7 Canada

patrick.brown@utoronto.ca 
Additional code

\section{European fertility}

URL's for web sites where data are obtained:

nutsUrl <- "http://ec.europa.eu/eurostat/cache/GISCO/geodatafiles/NUTS_2010_60M_SH.zip" nutsFile <- basename(nutsUrl)

Download and read in boundary file:

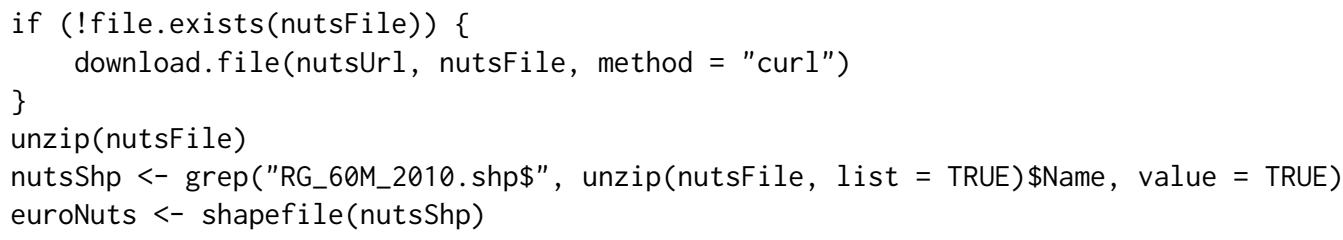

The fertility data are retrieved as a gzipped tab-separated text file, which the R.utils package is able to decompress. The code below will download a copy of this file from the author's web server if the EUROSTAT download fails.

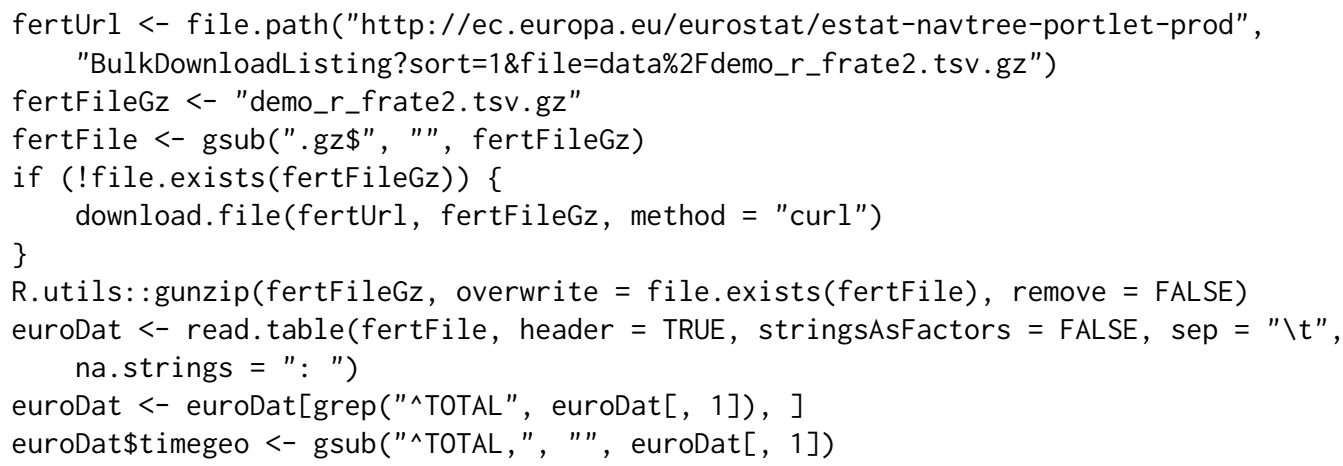

Merge the fertility and polygon data. Warning messages that not all rows of the fertility table can be matched to polygons can be ignored.

euroF <- sp: :merge(euroNuts, euroDat, all.x = FALSE, by.x = "NUTS_ID", by. $y=$ "timegeo")

Exclude some of the outlying parts of the EU:

euroF <- raster: :crop(euroF, extent $(-25,180,33,90)$ )

\section{Canada}

Transform the Great Circle:

gcircleT <- mapply (spTransform, CRSobj = crsList, MoreArgs = list $(x=$ gcircle $)$ )

Obtain background maps:

mapT <- mapply (openmap, crs = crsList, MoreArgs = list (path = "nrcan", $x=$ cities, buffer $=3$ ))

Create maps:

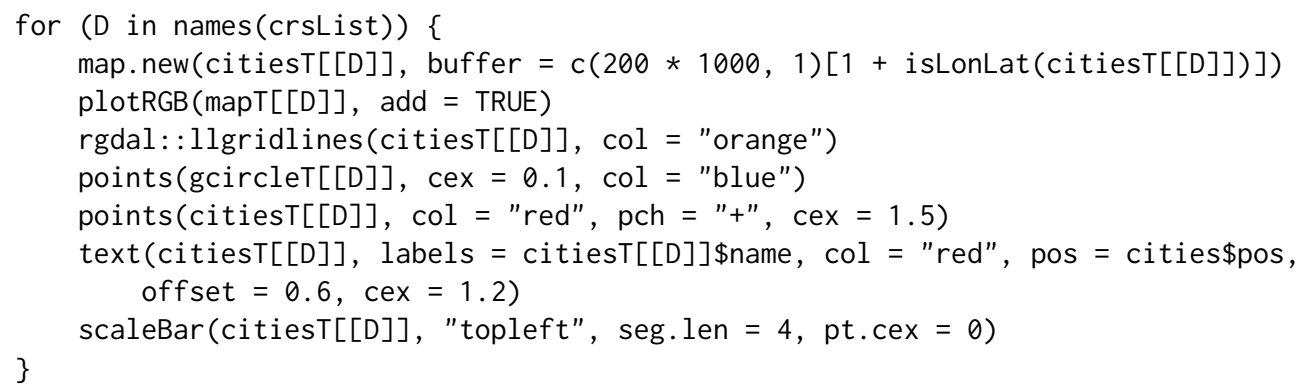




\section{Additional tables and figures}

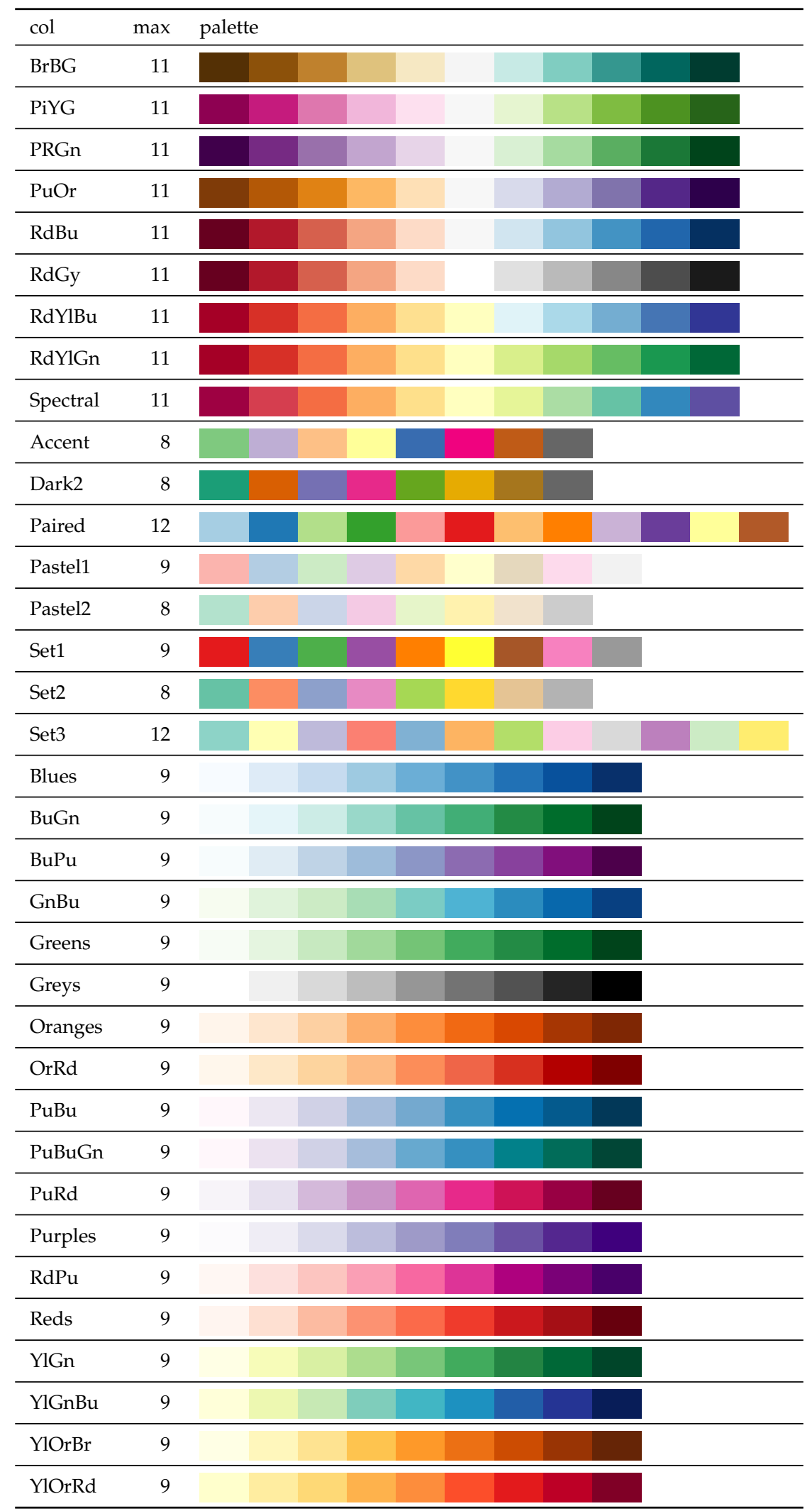

Table 4: Colour palettes from RColorBrewer, with col showing the character string to provide colourScale or brewer. pal and max giving the maximum number of colours for each palette. 


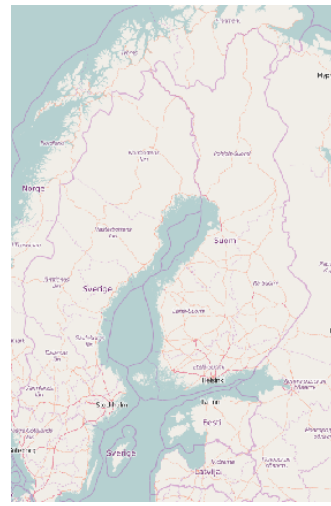

(a) osm,

(c) OpenStreetMap.

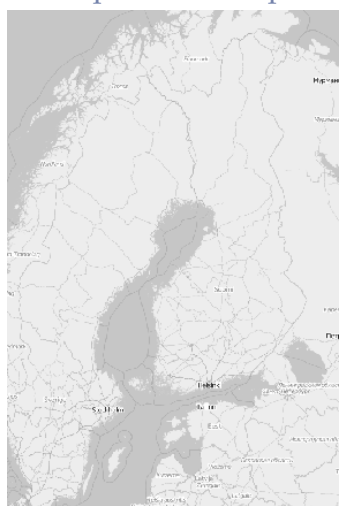

(e) bw-mapnik, (c) OpenStreetMap.

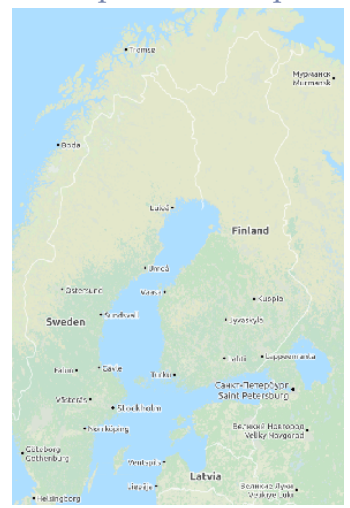

(i) landscape, (C) OpenStreetMap.

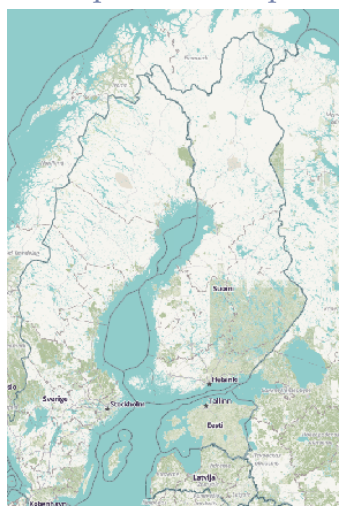

(m) humanitarian, (C) OpenStreetMap.

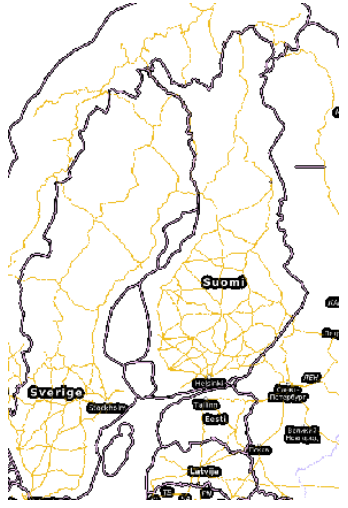

(b) osm-semitransparent, (C) OpenStreetMap.

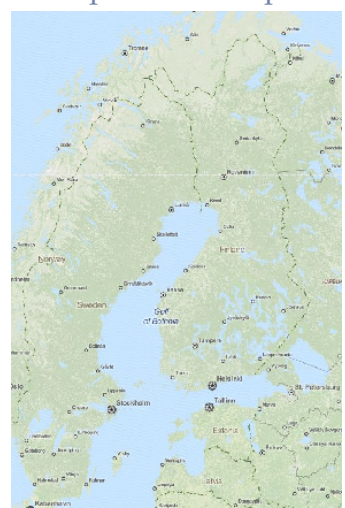

(f) mapquest. Tiles courtesy of MapQuest.

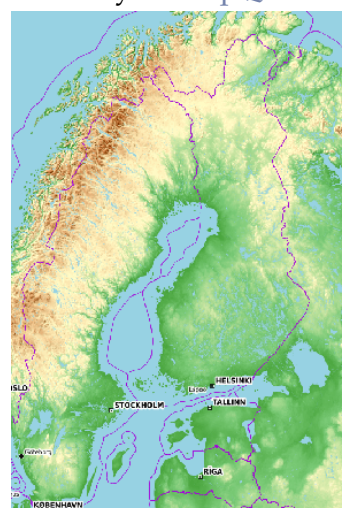

(j) opentopomap, (C) OpenStreetMap.

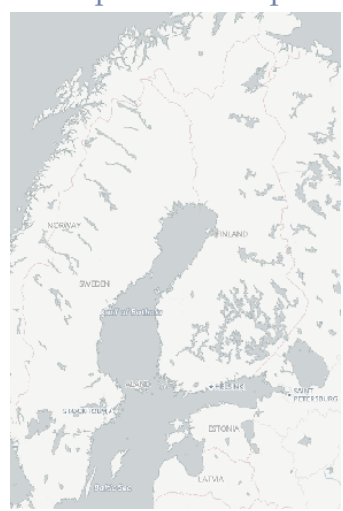

(n) cartodb, (c) CartoDB.

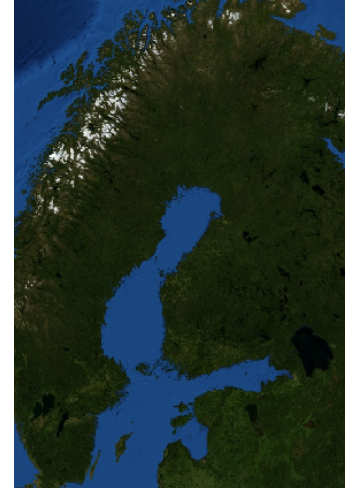

(g) mapquest-sat. Tiles courtesy of MapQuest.

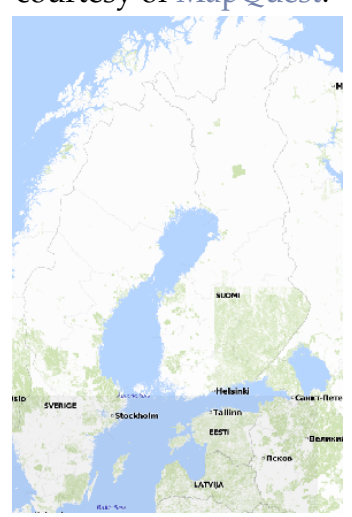

(k) maptoolkit, (C) Toursprung $\mathrm{GmbH}$

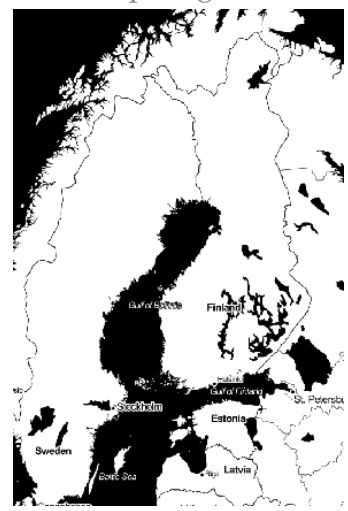

(o) stamen-toner, (c) Stamen Design.

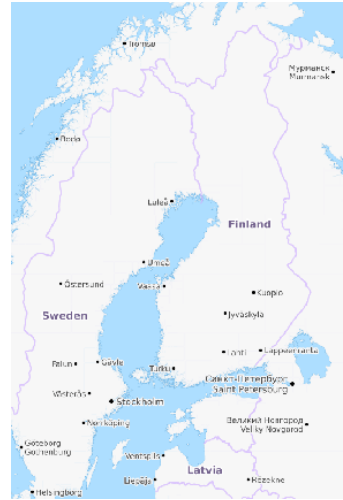

(d) osm-transport, (c) OpenStreetMap.

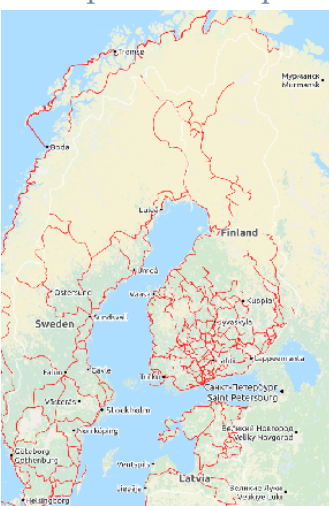

(h) osm-cyclemap, (c) OpenStreetMap.

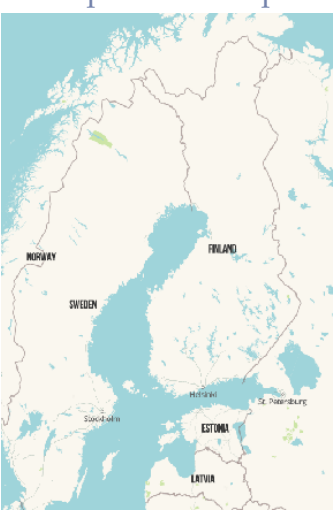

(l) waze, (c) Waze mobile.

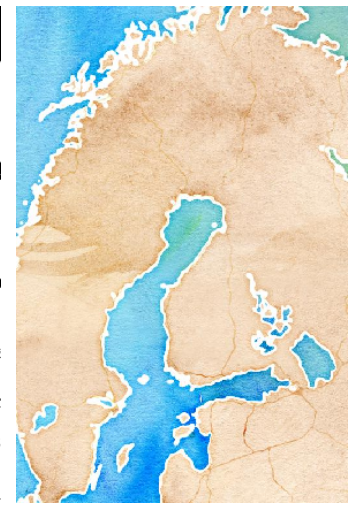

(p) stamen-watercolor, (c) Stamen Design.

Figure 11: Selected openmap tile sets. 